CERN-PPE/94-18

1 February 1994

\title{
ANTIHYDROGEN PHYSICS
}

M. Charlton ${ }^{1)}$, J. Eades $^{2)}$, D. Horváth ${ }^{3,4)}$, R. J. Hughes ${ }^{5)}$, C. Zimmermann ${ }^{6)}$

To be published in Physics Reports

1) University College, London, WC1E 6BT, UK

2) CERN, CH-1211 Genève, Switzerland

3) KFKI Research Institute for Particle and Nuclear Physics, $\mathrm{H}-1525$ Budapest, Hungary

4) INFN - Sezione di Pisa, I-56010 S. Piero a Grado, Pisa, Italy

5) Physics Division, Los Alamos National Laboratory, University of California, Los Alamos, NM 87545, USA

6) Max Planck Institute for Quantum Optics, Garching, Germany 


\section{Synopsis}

The hydrogen atom continues to be a testbench for a wide variety of fundamental physical measurements, whose scope has been expanded to the hydrogen-like atoms positronium and muonium. The hydrogen atom of antimatter, the bound state of an antiproton and a positron known as antihydrogen, has never been knowingly produced or studied. In this report, we discuss the production and spectroscopy of antihydrogen atoms, and describe the unique opportunities that such measurements would provide for tests of fundamental physical principles.

The principal difficulties in antihydrogen production are: collecting an adequate supply of the constituent particles (antiprotons and positrons); arranging for them to recombine efficiently into antihydrogen atoms, in spite of the low reaction rate; and controlling the produced atoms in a way that allows spectroscopic measurements to be performed on them. Developments in a number of different sub-fields of physics can now be brought to bear on these problems.

Substantial numbers of cryogenic antiprotons are regularly stored in Penning traps. Several methods are being considered for producing antihydrogen from antiprotons confined in these devices. In one of those, a second Penning trap with the correct field polarity to confine positrons is nested inside the outer trap containing antiprotons. The particle clouds intermingle inside the trap, and the slow recombination rate is compensated by the long confinement time. A second possible solution to the slowness of recombination is to inject a positron beam into the antiproton trap, creating positronium atoms at the trap wall; the cross-section for these atoms to react with the antiprotons to produce antihydrogen is very large. The positrons required can be obtained from radioactive sources or by electroproduction. Other production methods include: the stimulation of the radiative recombination from the $\mathrm{e}^{+}-\overline{\mathrm{p}}$ continuum with laser beams; using metastable antiprotonic states as $\bar{p}$ traps for the reaction with positron or positronium; and using pair-produced positrons from high-energy collisions of antiprotons with a gas jet target, thereby forming a $\mathrm{GeV}$ beam of antihydrogen atoms.

As the antihydrogen atoms are formed they must be cooled to remove the recombination energy and permit them to be trapped in a superimposed magnetic trap. Lyman- $\alpha$ lasers that could accomplish this are now being developed.

Great advances in the precision of hydrogen spectroscopy have come with laser developments that allow the study of sharp two-photon transitions, which are first-order Doppler free. Until recently, such measurements were performed on hydrogen atom beams containing numbers of atoms never likely to be attained with antihydrogen. However, the necessity to suppress the second-order Doppler effect has led to the use of cooled hydrogen beams, and spectroscopy on cryogenic hydrogen atoms, stored in the type of magnetic trap developed for Bose-Einstein condensation work, is now being pursued for even higher precision studies. This trapped atom technology could be applied to antihydrogen, because it would not require large numbers of atoms, and methods for the production of cold antihydrogen atoms and confining them in a magnetic trap are already under development.

The most intriguing transition that could be measured on the trapped antihydrogen atoms is the two-photon transition between the $1 S$ and $2 S$ states, which could be measured with a precision of $10^{-15}-10^{-18}$. This precision is possible because of the long 
lifetime $(1 / 8 \mathrm{~s})$ of the metastable $2 S$ level. A number of fundamental physical principles could be tested with this type of measurement. For instance, the Rydberg constant can be determined from the $1 S-2 S$ energy interval, and so by comparison of the Rydberg in hydrogen and antihydrogen, the first test of CPT for a fundamental atomic constant could be made, with a precision comparable to that given by the experimental limit on the difference of the neutral kaon masses. The antiRydberg can also be related to the more traditional CPT tests for particle properties. In combination with other measurements it can yield very high precision tests of CPT for the antiproton's charge and mass separately. This separation has not previously been accomplished with significant precision.

The $1 S-2 S$ measurement in antihydrogen can also provide the first test of the gravitational weak equivalence principle for antimatter, because the tranisition frequency is subject to a gravitational red-shift when moved through the same gravitational potential difference. When hydrogen and antihydrogen atoms are moved through a gravitational potential difference, their $1 S-2 S$ transitions will only red-shift equally if the atom and antiatom gravitational couplings are equal. The most easily used (and largest attainable) potential difference is the seasonal modulation of the solar potential at the surface of the earth. Because matter, and by inference hydrogen, is known to obey the equivalence principle, a constraint on the modulation of the hydrogen-antihydrogen $1 S-2 S$ frequency difference would provide a test of this principle for the positron, and the precision would be comparable to that for matter.

Antihydrogen production and experimentation will shortly be feasible. Antihydrogen spectroscopy will continue the tradition set with hydrogen and hydrogen-like systems such as positronium, muonium and exotic hydrogen atoms, of providing high precision tests of fundamental physical principles. 


\section{Table of Contents}

1. ANTIHYDROGEN - A FUNDAMENTAL PHYSICAL SYSTEM

Tests of CPT symmetry with antihydrogen

CPT invariance

CPT and antihydrogen

Tests of the weak equivalence principle for antiparticles

Antimatter and the equivalence principle

The gravitational red-shift

Experimental tests of WEP with antihydrogen

Other tests of the equivalence principle for antiparticles

2. PRODUCING THE CONSTITUENTS OF ANTIHYDROGEN

Antiprotons

Antiproton deceleration

Antiproton trapping and cooling

Positrons

Positron production

Positronium production

Positron trapping, cooling and bunching

3. MAKING THE ANTIHYDROGEN

Antihydrogen formation

Antihydrogen production by radiative recombination

Antihydrogen production in collisions of antiprotons with positronium

Antihydrogen production in $\overline{\mathrm{p}}+\mathrm{e}^{+}+\mathrm{e}^{+}$collisions

Antihydrogen production at $\mathrm{GeV}$ energies

Antihydrogen production using metastable antiprotonic helium states

Antihydrogen production in a combined $R F+$ Penning trap

Antihydrogen production: conclusion

Antihydrogen trapping and cooling

4. SPECTROSCOPY OF ANTIHYDROGEN

Spectroscopy from the ground state

One-photon $1 S \rightarrow 2 P$ transition

Two-photon $1 S \rightarrow 2 S$ transition

Spectroscopy from excited states

One-photon transition from $2 S$ to $3 P$ and $4 P$

Two-photon transition from $2 S$ to $4 S$

Transitions between Rydberg states

CONCLUSION

Spectroscopy during recombination

ACKNOWLEDGEMENTS

REFERENCES 
The concept of antimatter, which was introduced by Dirac between 1928 and 1931 [1, $2]$ has become so familiar that the production of antiprotons $(\bar{p})$ and positrons $\left(\mathrm{e}^{+}\right)$is regarded as quite routine. However, atomic antimatter has not yet been experimentally studied, largely because of the difficulties involved in producing and manipulating it. But recent progress in the trapping of charged and neutral particles, laser physics and in the theory of production mechanisms, makes it possible to consider seriously the production of and experimentation with the simplest anti-atom, which is the bound state of a positron and an antiproton known as antihydrogen $(\overline{\mathrm{H}})$ [3, 4]. Furthermore, the high precisions achieved in laser spectroscopy of hydrogen suggest that such techniques could be applied to antihydrogen to yield new tests of fundamental symmetries. (The new value of the hydrogen Rydberg produced by these techniques is now the most precise of any fundamental constant [5, 6] and even higher precisions are potentially attainable [7]). We discuss below the production and laser spectroscopy of antihydrogen with the principal objectives of developing new tests of CPT and the first direct tests of the weak equivalence principle (WEP) for positrons, with relevant, high levels of precision which would otherwise be unobtainable.

\section{Tests of CPT symmetry with antihydrogen}

\section{CPT invariance}

Dirac's prediction [2] of the existence of “...a new kind of particle, unknown to experimental physics, having the same mass and opposite charge to an electron..." (which was subsequently confirmed in Anderson's discovery of the positron [8]) shows the relationship between the properties of particles and their antiparticles that has since been systematized by the CPT theorem. This theorem, which was developed by Lüders [9], Pauli [10], Bell [11] and Jost [12], requires for its proof that a quantum field theory be constructed from fields that belong to finite-dimensional representations of the Lorentz group; have local interactions invariant under the proper Lorentz group; and be described by a hermitian Lagrangian. When these conditions are met, a quantum field theory will be invariant under the combined operations of $\mathrm{C}$ (charge-conjugation), $\mathrm{P}$ (parity) and $\mathrm{T}$ (time reversal). There is no such proof of invariance under each of these operations individually - the weak interactions are known to violate $\mathrm{C}, \mathrm{P}$ and $\mathrm{CP}$, and hence presumably also T. Thus, CPT invariance is the minimal condition for the existence of antiparticles within quantum field theory.

The action of the antiunitary CPT operator, $\Theta$, in quantum field theory is connected with reversal of the space-time coordinates, $x=x^{\mu}=(c t, \mathrm{x})$, as can be seen from its actions on a conserved vector current

$$
\Theta J^{\mu}(x) \Theta^{-1}=-J^{\mu}(-x)
$$

and the energy-momentum tensor

$$
\Theta T^{\mu \nu}(x) \Theta^{-1}=T^{\mu \nu}(-x) .
$$

Some of the implications of CPT invariance that can be deduced from these two 
equations are that a particle and its antiparticle have: equal and opposite additive quantum numbers, such as electric charge; and equal masses, total lifetimes and gyromagnetic ratios. A few of these predictions have been tested with high precision. An indirect test of the equality of the $\mathrm{K}^{0}$ and $\overline{\mathrm{K}}^{0}$ masses [13],

$$
\left|\frac{m\left(\mathrm{~K}^{0}\right)-m\left(\overline{\mathrm{K}}^{0}\right)}{m\left(\mathrm{~K}^{0}\right)}\right| \leq 5 \times 10^{-18},
$$

has set the scale of precision, but direct tests such as the equality of the electron and positron gyromagnetic ratios [14]

$$
\frac{g\left(\mathrm{e}^{+}\right)-g\left(\mathrm{e}^{-}\right)}{\left[g\left(\mathrm{e}^{+}\right)+g\left(\mathrm{e}^{-}\right)\right] / 2}=(-0.5 \pm 2.1) \times 10^{-12}
$$

and the equality of the proton and antiproton cyclotron frequencies in the same magnetic field [15],

$$
\left|\frac{\omega_{c}(p)-\omega_{c}(\bar{p})}{\omega_{c}(p)} \leq 4 \times 10^{-8}\right|,
$$

have significantly lower levels of precision. (Here, $\omega_{c}=e B / m$, is the cyclotron frequency of a particle of mass $m$ and charge $e$ in a magnetic field of strength $B$.)

However, the test of CPT in the neutral kaon system is based on an indirect and model-dependent connection between the measured quantities (properties of decay amplitudes) and the kaon mass matrix. Instead of associating any CPT violation with the masses of the neutral kaons, it could instead be attributed to the existence of a new, CPTviolating interaction. In this case the neutral kaon CPT test has a much less spectacular precision as the new interaction is only constrained to be less than $10 \%$ of the strength of the $\mathrm{CP}$-violating interaction [16]. The gyromagnetic and charge-to-mass ratio tests are direct measurements on particles in ion traps, but in the former, the measured quantity is the anomalous frequency (cyclotron - Larmor frequency difference). Three orders of magnitude in precision are 'gained' by expressing the comparison relative to the $g$-factor itself, rather than with respect to the anomaly, $(g-2)$.

The considerable physical content of modern metrology is not always widely appreciated, but precision measurements of the fundamental constants have provided some of the best tests of the laws of physics [17]. Precision tests of CPT for the static properties of elementary particles extend this metrological basis of physical theories into the realm of antiparticles. Thus, it is important to continue testing CPT precisely because it is so well-founded. At the same time one should remember that CPT invariance is not on such unshakable ground as, for instance, conservation of energy. The assumptions required to prove the theorem clearly show in which directions possible CPT violations might arise: Todorov et al. [18] have shown that CPT can be violated when non-finite dimensional representations of the Lorentz group are allowed; Wald [19] has argued that the curved space-time required by gravitation may be an obstacle to the proof of CPT (the proof requires flat space-time); and shortly before his death, John Bell developed a model field theory in which CPT violation occurred through non-locality. Yet another reason to test $\mathrm{CPT}$ is that it provides a link between the experimentally observed $\mathrm{CP}$-violation, and the as yet unobserved T-violation [20]. 
Figure 1: Energy levels of hydrogen and antihydrogen, showing: the principal structure from Bohr's (non-relativistic) quantum mechanics; the relativistic corrections from Dirac's theory which splits apart states of different angular momenta; the Lamb shift of quantum field theory which raises the energies of $S$-states; the hyperfine splitting ( $h f_{s}$ ) which arises from the magnetic interaction between the (anti)proton and the electron (positron); and the $1 S-2 S$ two-photon transition.

\section{CPT and antihydrogen}

The CPT symmetry of QED requires that the gross structure, fine structure and Lamb shifts, and the hyperfine structures in hydrogen and antihydrogen are identical (Fig. 1). Spectroscopic measurements of photon transition frequencies in antihydrogen could, in principle, be performed with very high precision, and comparison of these with the analogous transitions in hydrogen therefore tests CPT invariance with commensurate precision. In particular, the $1 / 8 \mathrm{~s}$ lifetime of the metastable $2 S$ level indicates an ultimate precision on measurements of the $1 S-2 S$ two-photon transition of $10^{-15}$ to $10^{-18}$, if the centre of the spectral line can be found to one part in $10^{3}$ and the line width can be reduced to the quantum limit [5]. Recently, an improvement of one order of magnitude (to 3.8 parts in $10^{11}$ ) on the precision of the value of the Rydberg was obtained from a measurement of this transition in hydrogen [6], and further improvements are expected [7]. If we assume that comparable precision will eventually be attained in the measurement of the Rydberg on antihydrogen, then we can anticipate a test of CPT for this fundamental atomic constant at the level of precision usually quoted in connection with the comparison of the neutral kaon masses.

The Rydberg in antihydrogen (anti-Rydberg), can be expressed in terms of the 
antiproton and positron charges and masses as

$$
R_{\overline{\mathrm{H}}}=\frac{m_{\overline{\mathrm{p}}} m_{\overline{\mathrm{e}}}}{m_{\overline{\mathrm{p}}}+m_{\overline{\mathrm{e}}}} \frac{\epsilon_{\overline{\mathrm{p}}}^{2} \epsilon_{\overline{\mathrm{e}}}^{2}}{8 \epsilon_{0}^{2} c h^{3}},
$$

where $e_{\overline{\mathrm{p}}}\left(m_{\overline{\mathrm{p}}}\right)$ and $\epsilon_{\overline{\mathrm{e}}}\left(m_{\overline{\mathrm{e}}}\right)$ are the charges (masses) of the $\overline{\mathrm{p}}$ and $\mathrm{e}^{+}$, respectively. One might think that the only fundamental antiparticle property that this expression could yield is a new value for the ratio of the positron and antiproton masses. However, there are other possibilities, which we can illustrate with a recently published example [21]. The expression for the Rydberg depends on the squares of the positron and antiproton charges, but no precision experimental tests of the relation of these charges to the charge quantum carried by the electron or proton are listed in the Review of Particle Properties [22]. In contrast, the equality of the electron and proton charge quanta is known with very high precision from experiments on the neutrality of matter [23]. A direct determination of the charge quantum of the antiproton and the positron would therefore be very fruitful from several points of view [21]:

1. Testing that a particle and its antiparticle have equal and opposite charges is as fundamental a test of CPT as testing the equality of their masses;

2. The electron-positron [24] and proton-antiproton [15] 'mass' comparisons from cyclotron frequency measurements remain comparisons of their charge to mass ratios until supplemented by direct measurements of the charges;

3. The measurement of the charge of the antiproton would extend the tests of charge quantization from electrons, protons and neutrons to antiparticles, with corresponding implications as a test of the quark model charge assignments;

4. The extension of charge tests to antiparticles will allow new tests of charge conservation, and hence electromagnetic gauge invariance to be inferred, or alternatively, it will permit the charges of other particles that cannot be measured directly (neutrinos) to be deduced;

5. A violation of charge quantization by antiparticles has been proposed as a mechanism for the cosmological separation of matter and antimatter [25].

The actual separation of the particles' charges and masses can be accomplished because the Rydberg involves the squares of their charges whereas their cyclotron frequencies are linear in them. To be specific, we introduce the electron-positron cyclotron frequency ratio [24]

$$
\rho_{\overline{\mathrm{e}}}=\frac{\omega_{c}(\mathrm{e})}{\omega_{c}(\overline{\mathrm{e}})},
$$

the electron-antiproton frequency ratio [15]

$$
\rho_{\overline{\mathrm{p} e}}=\frac{\omega_{c}(\mathrm{e})}{\omega_{c}(\overline{\mathrm{p}})},
$$

and the proton-antiproton frequency ratio [15]

$$
\rho_{\overline{\mathrm{p} p}}=\frac{\omega_{c}(\mathrm{p})}{\omega_{c}(\overline{\mathrm{p}})},
$$

where $\omega_{c}=\frac{q B}{m}$ is the cyclotron frequency of a particle of charge $q$ and mass $m$ in a magnetic field $B$. Using these quantities the antihydrogen Rydberg can be re-expressed 


$$
\frac{R_{\overline{\mathrm{H}}}}{R_{\mathrm{H}}}=\frac{\frac{m_{\mathrm{p}}}{m_{\mathrm{e}}} \frac{\rho_{\overline{\mathrm{p}} e}}{\rho_{\overline{\mathrm{p}} \mathrm{p}}}}{\rho_{\overline{\mathrm{p}} \mathrm{e}}\left(\frac{\epsilon_{\bar{e}}}{e}\right)+\rho_{\overline{\mathrm{e} e}}\left(\frac{e_{\overline{\mathrm{p}}}}{e}\right)}\left(\frac{e_{\overline{\mathrm{p}}}}{e}\right)^{3}\left(\frac{e_{\overline{\mathrm{e}}}}{e}\right)^{3},
$$

where $e$ is the quantum of charge of the electron or proton and $R_{\mathrm{H}}$ is the hydrogen Rydberg. This equation now relates the antihydrogen Rydberg and other measurable quantities to the antiproton and positron charges. To extract an expression for the antiproton charge alone we can use a separate equation to express the positron charge in terms of the positronium Rydberg, $R_{\mathrm{Ps}}$ [26], and the electron-positron cyclotron frequency ratio:

$$
\left(\frac{e_{\overline{\mathrm{e}}}}{e}\right)^{3}-\left(\frac{R_{\mathrm{Ps}}}{2 R_{\infty}}\right)\left(\frac{e_{\overline{\mathrm{e}}}}{e}+\rho_{\overline{\mathrm{e}} \mathrm{e}}\right)=0,
$$

where $R_{\infty}=m_{\mathrm{e}} e^{4} /\left(8 \epsilon_{0}^{2} c h^{3}\right)$ is the Rydberg of hydrogen for infinite proton mass.

Substituting the experimental value for $e_{\overline{\mathrm{e}}} / e$ from (11) to (10) yields an expression for $e_{\overline{\mathrm{p}}} / e$ in terms of measured quantities. The various cyclotron frequency ratios and the positronium Rydberg have all been measured with precisions of a few parts in $10^{8}$, but there are proposals to improve the precision of the cyclotron frequency comparison to the level of one part in $10^{11}$ [27], which is also the ultimate precision of the $1 S-2 S$ interval from which the positronium Rydberg is determined [28]. If these hopes are realized, and the antihydrogen Rydberg could be measured with the precision discussed earlier, a test of the equality of the electron and antiproton charge quanta at the one part in $10^{11}$ level could be deduced. This precision exceeds by at least 5 orders of magnitude the test that can be inferred from measurements of the Rydberg in antiprotonic atoms using similar arguments [21]. (It was because the precision of such measurements was limited that the cyclotron frequency measurement in an ion trap was developed.)

A second example of the application of antihydrogen spectroscopy to CPT tests for antiparticle properties would involve the measurement of the $n=1$ level hyperfine structure, from which the ratio of the antiproton's gyromagnetic ratio to that of the bound positron could be deduced. An experiment of this type has been performed on hydrogen, where allowing for a small QED correction to the electron's gyromagnetic ratio, the measured quantities can be related to the proton's magnetic moment measured in units of Bohr magnetons [29]. The precision attained on this quantity for the proton was about 1 part in $10^{8}$, whereas the magnetic moment is now known much more precisely from other experiments involving NMR on bulk quantities of water. In contrast, the antiproton's magnetic moment is only known to a precision of about 3 parts in 1000 from the hyperfine structure of antiprotonic atoms [30]. If the precision of the hydrogen measurements could be reproduced on antihydrogen, the test of CPT for the proton and antiproton magnetic moments could be improved by about 5 orders of magnitude. (An ion trap measurement of the antiproton magnetic moment is being prepared [31]. However, the smallness of the nuclear magneton suggests that the high precisions attained in the electron and positron $g$-factor measurements are not likely to be repeated.)

It is important to contrast the CPT tests that could be made on antihydrogen with those possible on other systems. The anticipated improvements in the precision of ion trap cyclotron frequency measurements to one part in $10^{11}$ is still many orders of magnitude less than potentially attainable with the $1 S-2 S$ antihydrogen transition. Moreover, we have also seen that different properties of the antiparticles are probed by the two types 
of measurement. Precision measurements of the $1 S-2 S$ two-photon transition have also been made in positronium [26] and muonium [32]. But again, the precisions ultimately possible are many orders of magnitude lower than with antihydrogen, and the constituents are also purely leptonic, so that they could not provide CPT tests for baryonic properties.

\section{Tests of the weak equivalence principle for antiparticles}

In Newtonian gravitation the acceleration, $g$, of a body in the Earth's gravitational field can be expressed as

$$
m_{i} g=m_{g} \frac{G M_{E}}{r^{2}}
$$

where $G$ is Newton's constant, $M_{E}$ is the mass of Earth, $r$ is the distance of the body from the centre of Earth, and $m_{i}\left(m_{g}\right)$ are the body's inertial (gravitational) masses, respectively.

The experimental observation that materials of different compositions fall with the same acceleration in the same gravitational field (universality of free-fall) acquired a deep theoretical significance through Einstein's formulation of the equivalence principle [33]. According to this principle, an observer in free-fall "... has the right to consider his state as one of rest and his environment as field-free relative to gravitation." [34]. This principle is a cornerstone of general relativity, and more generally, all metric theories of gravity. Different versions of the equivalence principle, restricting its application to different classes of phenomena, are now in use [35]. The weak equivalence principle (WEP) limits attention to mechanical quantities, whereas the Einstein equivalence principle (EEP) includes WEP and all non-gravitational phenomena. (Schiff conjectured that WEP actually implies EEP because the mass of an atom includes contributions from various types of energy, not just the rest masses of the constituents [36].)

Tests of WEP began with the apocryphal experiments of Galileo, but only achieved a significant level of precision with the Eötvös experiment [37] at the end of the last century. Recently, new versions of this experiment have shown that different types of matter fall with the same accelerations with a precision approaching one part in $10^{11}$ [38]. However, WEP has never been tested directly for the commonly available antiparticles: antiprotons and positrons. (There are very few empirical WEP tests for any elementary particles, although the neutron and the electron plus proton can be inferred to obey WEP with precisions of one part in $10^{8}$ from the results of Eötvös experiments $[39,40]$.)

\section{Antimatter and the equivalence principle}

The notion that antimatter might behave differently from matter in a gravitational field dates back to Maxwell [41], who briefly considered if the theory of electrodynamics (which contained the inverse-square law) could also be applied to gravity. However, because he was dealing with what would nowadays be called a vector theory, he found that to replace the "likes repel" law of electrostatics with a gravitational attraction, he would need to allow the field energy to become negative. The problems that this would lead to caused Maxwell quickly to dismiss this idea. Other authors were not so easily discouraged. Both Lorentz [42] and Heaviside [43] pursued this idea, and Schuster [44] made the now obvious extrapolation: " if there is negative electricity, why not negative gold?" i.e. an opposite kind of matter, that would fall up. This primitive notion of anti-atoms is prob- 
ably the origin of the strange idea that antimatter might be gravitationally repelled by matter. Today it is known that vector components of gravity are very strongly excluded, and that the experimental data are consistent with a tensor gravitational interaction. A vector-scalar modification of gravity [45] that would have caused a larger gravitational interaction of the antiproton is likewise strongly constrained [39, 46]. Nevertheless, these results leave WEP and the tensor gravitational coupling to antiparticles untested.

The question of the gravitational behaviour of antimatter was again considered during the few years after the discovery of the antiproton, and several arguments were developed for compliance with the WEP. Two of these are quantitative but indirect, whereas the rest involve other physical principles, and so it is to these that we turn first. One argument suggests that an anomalous gravitational acceleration of the antiproton would violate CPT symmetry. However, it must be remembered that a gravitating particle is not isolated, so that its gravitational acceleration is not an intrinsic property. Instead, the gravitational acceleration involves the interaction of the particle with the source of the gravitational field, which in this case is the earth. Therefore, when properly applied, CPT would require that the gravitational acceleration of an antiproton in the field of an anti-earth would be equal to that of proton in the field of our earth. Thus, CPT alone cannot relate the gravitational acceleration of matter to that of antiparticles.

\section{The gravitational red-shift}

Morrison developed an argument, which invokes conservation of energy and the gravitational red-shift [47]. It is based on a gedanken experiment developed by Einstein to show that, because of the weight of energy, identical clocks will run at different rates at different gravitational potentials [48]. (Here, a clock is some local system with a precise frequency that can be used to measure proper time). We start by reviewing a generalization of Einstein's argument [49, 50].

We consider a local clock based on the photon frequency in the transition $A^{*} \rightarrow A+\gamma$, between a system $A$ and its excited state, $A^{*}$. In a uniform gravitational field $A\left(A^{*}\right)$ has gravitational acceleration $g\left(g^{*}\right)$ and inertial mass $m\left(m^{*}\right)$, respectively. Einstein's dumb waiter device [48] (a mechanical system of belts and pulleys with trays at upper and lower levels on which objects may be placed) can be used to compare the relative rates of two such clocks at different levels in the gravitational field by exchanging photons. The device is prepared with system $A$ located at the lower level, and system $A^{*}$ at a level $l$, above (see Fig. 2a).

The device allows us to interchange $A$ and $A^{*}$, with the output of an amount of mechanical energy (Fig. 2b),

$$
E_{\text {out }}=\left(m^{*} g^{*}-m g\right) l,
$$

which equals the extra gravitational potential energy of the excited state. System $A^{*}$ is now located at the lower level and is allowed to decay to its ground state, $A$, by emission of a photon of frequency $\omega_{1}$, with $\hbar \omega_{1}=\left(m^{*}-m\right) c^{2}$, which defines the local clock frequency. We arbitrarily measure all gravitational potential energies with respect to the lower level.

This photon is allowed to propagate to the upper level. During its propagation its total energy does not change: the gravitational field is assumed to be static; there is no dissipation; and we assume energy conservation. Thus, when it arrives at the upper level it has insufficient energy to excite the system $A$ to $A^{*}$ because of the extra gravitational 
Figure 2: Einstein's dumb waiter device. (a) Initial state; (b) after changing positions in the gravitational field; (c) after photon exchange.

potential energy required to make the transition at that level. This shortfall can be expressed in terms of the energy of the transition, and hence the local frequency, $\omega_{2}$, of the corresponding photon that would be emitted at the upper level as

$$
\hbar \omega_{2}=\hbar \omega_{1}\left(1+g_{r} l / c^{2}\right),
$$

where $g_{r}$, which has the dimension of acceleration, parameterise the generalized red-shift, $\Delta \omega / \omega=g_{r} l / c^{2}$, of the clock frequency with height.

In order to excite the system $A$ at the upper level and restore the whole device to its starting configuration, the energy carried by the photon must therefore be augmented by an amount (Fig. 2c)

$$
E_{\text {in }}=\left(m^{*}-m\right) g_{r} l
$$

Conservation of energy requires $E_{\text {in }}=E_{\text {out }}$, and hence

$$
g_{r}=\frac{m^{*} g^{*}-m g}{m^{*}-m}=\frac{\Delta(\text { clock weight })}{\Delta(\text { clock mass })} .
$$

From Equation (16) we see that, as a consequence of energy conservation, there will be some red-shift as long as the energy content of the clock contributes to the clock's weight, and hence has gravitational potential energy. (Moreover, clocks based on different transition energies could red-shift by different amounts if their energies make different contributions to their weights.) A test of WEP for the energy content of the clock system could be obtained by comparing the rate of two identical clocks at two different gravitational potentials [49]. It is only when WEP is obeyed, $g^{*}=g$, that the conventional red-shift, $g_{r}=g$ - and hence $\Delta \omega / \omega=\Delta U / c^{2}$, where $U$ is the Newtonian gravitational potential - is obtained. 
This argument is possibly one of the greatest sources of confusion and misinterpretation in gravitational physics. It is the energy levels of the clock that change with height in the gravitational field because of their gravitational potential energy. The only role of the photon is to transfer energy and hence frequency information from one place to another in a gravitational field, which could be accomplished by a variety of other means. This is a point that has been elucidated particularly well by Bell [51]. Moreover, there is a red-shift in theories of gravity, such as Nordström's scalar theory, in which light has no weight [52].

In Morrison's argument [47] the clock frequency is not an atomic transition, but is based on the photon frequencies in electron-positron annihilation. Using, once again, Einstein's dumb waiter device, we load an electron and a positron (the system $A^{*}$ ), each of which has rest mass $m_{e}$, onto the device at the upper level, leaving the lower tray empty (the system A). The gravitational acceleration of the $\mathrm{e}^{+}\left(\mathrm{e}^{-}\right)$is $\bar{g}(g)$, respectively. The device is now used to lower the $\mathrm{e}^{+} \mathrm{e}^{-}$pair with the output of an amount of energy,

$$
E_{\text {out }}=m_{\mathrm{e}}(\bar{g}+g) l,
$$

At the lower level the $\mathrm{e}^{+} \mathrm{e}^{-}$pair is allowed to annihilate into photons with combined frequencies $\omega_{1}$, with $\hbar \omega_{1}=m_{\mathrm{e}} c^{2}$, constituting the local clock. The photons are directed back up to the upper level, but again have insufficient energy to recreate an electronpositron pair, because of the extra gravitational potential energy of a pair at this level. Therefore, according to a local $\mathrm{e}^{+} \mathrm{e}^{-}$-annihilation clock at the upper level of frequency $\omega_{2}$, their frequencies have been red-shifted according to

$$
\hbar \omega_{2}=\hbar \omega_{1}\left(1+g_{r} l / c^{2}\right),
$$

Here, again, $g_{r}$ expresses the generalized red-shift of this clock system. Thus, if augmented with an amount of energy,

$$
E_{\text {in }}=2 m_{\mathrm{e}} g_{r} l,
$$

the photons' energy can be used to re-create the $\mathrm{e}^{+} \mathrm{e}^{-}$pair in the original configuration of the device. Conservation of energy requires $E_{\text {in }}=E_{\text {out }}$, or

$$
g_{r}=\frac{\bar{g}+g}{2}=\frac{\text { pair weight }}{\text { pair mass }}
$$

as before. Thus, Morrison's argument is merely a special case of Einstein's original argument.

When this argument was first constructed it was believed (erroneously) that the redshift was a property of the photon (weight of light), and that therefore the annihilation photons would red-shift by exactly the same amount as did the gamma-ray photons in the Pound-Rebka [53] experiment: i.e. $g_{r}=g$, and hence the positron would have to obey WEP. As we saw above, the red-shift reflects the extent to which the energy content of clocks [50, 51, 54] obeys the equivalence principle: it has nothing whatsoever to do with a property of the photon [55]. An anomalous gravitational acceleration of the antiparticle $(\bar{g} \neq g)$ is entirely consistent with conservation of energy [56, 57], at the expense of an anomalous red-shift $\left(g_{r} \neq g\right)$ of the annihilation photons [58, 59], i.e. the relative rate (red-shift) of annihilation clocks that are separated in height by $l$ is

$$
\Delta \omega / \omega=\frac{g+\bar{g}}{2} \frac{l}{c^{2}} \neq \Delta U / c^{2},
$$


where $\Delta U=g l$ is the Newtonian potential difference. Conversely, the measurement of $g_{r}$ determines the positron's gravitational acceleration, $\bar{g}$, and hence tests WEP. This analysis shows that the often- made assertion that a violation of WEP by antimatter engenders a failure of energy conservation is incorrect.

\section{Experimental tests of WEP with antihydrogen}

Several authors have proposed that the gravitational acceleration of the antihydrogen atom could be measured directly [60,61], thereby providing a WEP test for the antiproton. This method for testing WEP has the virtue of dealing with a neutral particle, and would therefore be free of troublesome systematic errors due to stray electric fields. Nevertheless, experience from other experiments that measure the gravitational accelerations of neutral particles or atoms suggests that high precisions are unlikely to be attained [62]. Here it should be pointed out that the misconception persists that Witteborn and Fairbank's attempt [63] to compare the free-fall acceleration of the positron with that of the electron was successful, and that further experiments on falling fundamental antiparticles are therefore redundant. In fact, these workers were only able to determine a value for the gravitational acceleration of the electron. This measurement had an accuracy of only $10 \%$, and it was never satisfactorily explained why the stray electric fields in their drift tube resulted in the value found. The drift-tube technique has now been adopted by the Los Alamos group [66] in an attempt to measure the gravitational acceleration of the antiproton. The influence of the stray fields on the antiproton should be 1840 times smaller, but even so the precision expected from the measurement, if successful, will not be much better than $1 \%$.

We saw above that the measurement of a red-shift can be interpreted as a test of WEP [49], but because the red-shift is a relativistic gravitational effect, and is therefore small (at the Earth's surface), an absolute test of WEP for the positron based on the red-shift of the annihilation clock would be extremely difficult [40]. However, other clocks based on positrons can be used instead, and a relative red-shift experiment based on the $1 S-2 S$ transitions in hydrogen and antihydrogen offers the prospect of very good tests of WEP for antimatter because of the precision of spectroscopic measurements [59]. Because the $1 S-2 S$ binding energy difference in antihydrogen has a positron kinetic energy component (for which we wish to derive a test of WEP) and an electrostatic potential energy component (which is known to obey WEP from the results of Eötvös experiments), we must consider how gravity couples to these quantities. We start by reviewing the effects of conventional gravity on the hydrogen atom, which is known to obey WEP.

The $1 \mathrm{~S}$ and $2 \mathrm{~S}$ energy level difference in hydrogen

$$
\Delta E(1 S-2 S)=\frac{3 m_{\mathrm{e}} e^{4}}{8 \hbar^{2} c^{2}}
$$

(in Bohr approximation), is derived from the Schrödinger equation of the electron in the Coulomb field of the proton. The electron mass instead of the reduced mass appears in this equation for simplicity. Conventional gravity can be incorporated by introducing the interaction Lagrangian density

$$
L_{\mathrm{int}}=-\frac{1}{2} h_{\mu \nu} T^{\mu \nu}
$$


where

$$
h_{\mu \nu}=\frac{2 U}{c^{2}} \operatorname{diag}(1,1,1,1)
$$

is the tensor gravitational field at the surface of the Earth, $U$ is the Newtonian gravitational potential, and $T^{\mu \nu}$ is the energy- momentum tensor of the electron and the Coulomb field in the hydrogen atom. We use the convention in which the metric of flat space-time is $\eta^{\mu \nu}=\operatorname{diag}(1,-1,-1,-1)$.

The modification to the Schrödinger equation can be deduced from the corresponding gravitationally modified action for an electron of mass $m_{\mathrm{e}}$ in an electromagnetic field. The relevant terms are contained in the particle action

$$
S \approx \int d t\left[m_{\mathrm{e}} c^{2}\left(1+\frac{U}{c^{2}}\right)+\frac{1}{2} m_{\mathrm{e}} v^{2}\left(1-\frac{3 U}{c^{2}}\right)\right],
$$

where $v$ is the particle's speed and we have assumed $\frac{v^{2}}{c^{2}},\left|\frac{U}{c^{2}}\right| \ll 1$, and the action of the electromagnetic field

$$
S_{\mathrm{em}}=\frac{1}{8 \pi c} \int d^{4} x\left[\left(1-\frac{2 U}{c^{2}}\right) \vec{E}^{2}-\left(1+\frac{2 U}{c^{2}}\right) \vec{B}^{2}\right] .
$$

There are no gravitational terms in the action for the interaction of the charged particle and the electromagnetic field.

From Eq. (25) we see that in these flat space-time coordinates we must make the inertial mass replacement [64]

$$
m_{\mathrm{e}} \rightarrow m_{\mathrm{e}}\left(1-\frac{3 U}{c^{2}}\right)
$$

in the Schrödinger equation. Similarly, from Eq. (26) we see that the gravitational field acts like a medium with permeability and permittivity [64]

$$
\mu=\epsilon=\left(1-\frac{2 U}{c^{2}}\right)
$$

so that we must also make the replacement

$$
e^{2} \rightarrow e^{2}\left(1+\frac{2 U}{c^{2}}\right)
$$

giving the Schrödinger Hamiltonian, $H$, in the presence of gravity

$$
H=-\left(1+\frac{3 U}{c^{2}}\right) \frac{\hbar^{2}}{2 m_{\mathrm{e}}} \nabla^{2}-\left(1+\frac{2 U}{c^{2}}\right) \frac{e^{2}}{r},
$$

where $\mathrm{U}$ is to be treated as a constant over the dimensions of the atom.

The net result for hydrogen is then [59]

$$
\Delta E(1 S-2 S) \rightarrow \Delta E(1 S-2 S)\left(1+\frac{U}{c^{2}}\right)
$$

showing, as expected, that the $1 S-2 S$ energy difference possesses a weight in proportion to its mass. Moreover, because the frequencies of the photons emitted in this transition 
are related to the energy difference through Planck's constant, this equation also shows that this clock experiences the normal red-shift: $\Delta \omega / \omega=\Delta U / c^{2}$.

Although it is difficult to develop a consistent field theory in which antimatter does not obey WEP, it is possible to consider a violation within a phenomenological classical or quantum mechanical model that will be adequate for our purposes. Because we know from Eötvös experiments that normal bulk matter and electromagnetism respect WEP to very high accuracy, we will assume that they experience conventional gravity through the interaction of Eq. (23). But we will allow a violation of WEP by assuming that the usual, essentially infinite-range tensor gravitational field, $h_{\mu \nu}$, couples to the energy-momentum tensor of antimatter with an anomalous strength. Therefore, we allow positrons and antiprotons to experience an anomalous gravitational interaction by introducing the Lagrangian density $[57,58,59]$

$$
L_{\mathrm{int}}=-\frac{1}{2} \alpha_{g} h_{\mu \nu} T^{\mu \nu}
$$

In Eq. (32) $T^{\mu \nu}$ is the energy-momentum tensor of the antiparticles, and $\alpha_{g}$ is an adjustable coupling parameter between antimatter and the gravitational field.

A deviation of $\alpha_{g}$ from unity reflects a violation of WEP, so that if ordinary matter experiences gravitational acceleration $g$, antimatter will experience an anomalous acceleration, $\bar{g}=\alpha_{g} g$ [58]. Different $\alpha_{g}$ parameters should be introduced for the various an-

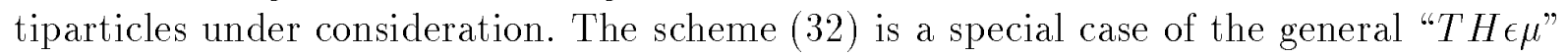
formalism that has been developed to analyze the rôle of the equivalence principle in the coupling of gravity to electromagnetic systems [35].

For the $1 S-2 S$ energy interval in antihydrogen we must make the inertial mass replacement

$$
m_{\mathrm{e}} \rightarrow m_{\mathrm{e}}\left(1-\frac{3 \alpha U}{c^{2}}\right)
$$

instead of Eq. (27), in the Schrödinger equation for the positron in the electrostatic field of the antiproton, with the result [59].

$$
\Delta \bar{E}(1 S-2 S) \rightarrow \Delta \bar{E}(1 S-2 S)\left[1+(3 \alpha-2) \frac{U}{c^{2}}\right]
$$

where the bar denotes a transition in antihydrogen. This equation shows that the $1 S-2 S$ energy difference in antihydrogen has an anomalous gravitational potential energy if the positron violates WEP, and hence that this transition frequency has an anomalous redshift.

The fractional frequency difference between $1 S-2 S$ transition photons from hydrogen and antihydrogen at the same height is then

$$
\frac{\bar{\omega}(1 S-2 S)-\omega(1 S-2 S)}{\omega(1 S-2 S)}=3(\alpha-1) \frac{U}{c^{2}}
$$

Thus, any difference that arises between the $1 S-2 S$ transition frequencies in hydrogen and antihydrogen as both are moved through the same gravitational potential difference, $\Delta U$,

$$
\frac{\Delta \bar{\omega}(1 S-2 S)-\Delta \omega(1 S-2 S)}{\Delta \omega(1 S-2 S)}=3(\alpha-1) \frac{\Delta U}{c^{2}}
$$


would be indicative of a violation of WEP by antihydrogen [59]. This concept is known as a null red-shift experiment [35, 65].

The largest potential difference that can be produced requires no motion with respect to the earth, because the eccentricity of the Earth's orbit around the sun produces a change of the solar potential of $\frac{\Delta U}{c^{2}}=3 \times 10^{-10}$ during a three-month period. A null red-shift experiment performed with a precision of one part in $10^{18}$ could therefore test WEP for the positron with a precision of one part in $10^{9}$ using the potential difference introduced above [59]. Using the reduced mass instead of the positron's mass, WEP for the antiproton could also be tested to a precision of five parts in $10^{5}$. (The level of precision would be reduced by three orders of magnitude if the diurnal variation of the solar potential was used instead.) So the null red-shift experiment would directly test WEP for the positron with the same precision as it has been tested for the constituents of ordinary matter $\left(\left|\alpha_{g}-1\right|<10^{-8}\right)$.

\section{Other tests of the equivalence principle for antiparticles}

Other particle-antiparticle frequency comparisons are not capable of providing such precise tests. The electron-positron cyclotron frequency comparisons, even with their projected future precision $\left(10^{-11}\right)$, would only be able to test WEP for the positron at the $1 \%$ level by constraining any seasonal modulation of their relative frequencies. We should also compare the significance of an antihydrogen null red-shift experiment to the two indirect quantitative arguments developed in the late 1950s.

Schiff [67] argued that virtual positrons contribute to both the mass and the weight of atoms, and that therefore the Eötvös experiments would have revealed any WEP violation for these antiparticles. His calculations imply that the inertial - gravitational mass difference of an atom of atomic number $Z$ is

$$
m_{i}-m_{g}=\left(\alpha_{g}-1\right) \frac{4 m}{3 \pi^{2}}\left(\frac{Z}{137}\right)^{2}\left[\log \left(\frac{h}{m c R_{\text {nuc }}}\right)+0.338\right]
$$

where $\alpha_{g}$ parameterise a violation of WEP by the positron's rest mass, and $R_{\text {nuc }}$ is the nuclear radius. When this calculation is confronted with the latest experimental data [38],

$$
\left|\frac{g(B e)-g(C u)}{g}\right|<10^{-11},
$$

it suggests that the positron obeys WEP with a precision of one part in $10^{6}$, (i.e. $\mid \alpha_{g}-$ $1 \mid<10^{-6}$ ), which is two orders of magnitude less than the precision of the antihydrogen test suggested above. However, on closer inspection [68], Schiff's calculation involves a gravitational coupling that is not consistent with the known (tensor) nature of this field, nor with any field theory that respects Lorentz invariance. This is because Schiff allowed the positron's rest mass to violate WEP but required its inertial mass (kinetic energy) to respect WEP. We conclude that, although quantitative, Schiff's calculation cannot be regarded as definitive because of its conflict with known properties of the gravitational interaction.

Another argument was developed by Good[69], which is in effect a variant of the red-shift discussion above. The argument can be applied to any clock (of frequency $\omega$ ) and the CPT-conjugate anticlock (of frequency $\bar{\omega}$ ) that are required to have identical 
frequencies, $\omega=\bar{\omega}$, by CPT symmetry in the absence of gravity (at infinity, where the physically motivated boundary condition, $U \rightarrow 0$, must be imposed $[35,69,59])$. Then if the anticlock violates WEP, there will be, in addition to diurnal and seasonal modulations of their relative frequencies, a constant frequency offset owing to the anomalous red-shift of the WEP-violating clock between infinity and the surface of the earth. This offset is apparent in Eq. (35) for the difference of the hydrogen and antihydrogen $1 S-2 S$ transitions at the same gravitational potential. The value of the gravitational potential at the surface of the Earth, calculated according to Poisson's equation, increases as the effects of matter at greater and greater distances are introduced [69] (the largest value that can be estimated from astronomical observations and the inverse-square law is the potential of the local galactic supercluster [71]: $U$ (supercluster) $/ c^{2}=3 \times 10^{-5}$ ). Thus, a very strong constraint on WEP can be deduced if the frequencies are measured to be equal.

Good applied this argument to the de Broglie clocks of the $\mathrm{K}^{0}$ and $\overline{\mathrm{K}}^{0}$ particles of frequencies $\omega=m\left(\mathrm{~K}^{0}\right) c^{2} / \hbar$ and $\bar{\omega}=m\left(\overline{\mathrm{K}}^{0}\right) c^{2} / \hbar$, respectively. The argument has since been strengthened [71, 70,72], and linked to WEP for the antiproton via the quark model [72]. In the absence of gravity, the neutral kaon wave function at time $t$, corresponding to a $\mathrm{K}^{0}$ created at $t=0$ may be described by [73]

$$
\psi(t)=\exp \left[i S_{c l}(t) / \hbar\right] \psi\left(\mathrm{K}^{0}\right)
$$

where the $\mathrm{K}^{0}$ 's wave function is $\psi\left(\mathrm{K}^{0}\right)=\left(\begin{array}{l}1 \\ 0\end{array}\right)$, the $\overline{\mathrm{K}}^{0}$ 's is $\psi\left(\overline{\mathrm{K}}^{0}\right)=\left(\begin{array}{l}0 \\ 1\end{array}\right)$, and the action is

$$
S_{c l}=-\int_{0}^{t} d t^{\prime} M \frac{c^{2}}{\gamma}
$$

In Eq. (40) $\gamma=\left(1-v^{2} / c^{2}\right)^{-1 / 2}$, where $v$ is the speed of the kaon, the mass matrix is

$$
\mathrm{M}=\left(\begin{array}{cc}
m & \Delta m / 2 \\
\Delta m / 2 & m
\end{array}\right)
$$

where $m$ is the mass of the $\mathrm{K}^{0}$ and $\overline{\mathrm{K}}^{0},\left(m_{1,2}=m \mp \Delta m / 2\right.$ are the masses of the $\mathrm{K}_{1,2}=$ $\left(\mathrm{K}^{0} \pm \overline{\mathrm{K}}^{0}\right) / \sqrt{2}$ propagation eigenstates, respectively) and the integration is performed along the particle's trajectory. We ignore the possible CP- or CPT-violating terms, and the imaginary parts in M, because these will not be relevant in what follows. The effect of an anomalous gravitational interaction on this system can be introduced in the same way as for antihydrogen.

The matrix analogue of the energy-momentum tensor for the neutral kaons is

$$
T^{\mu \nu}=\mathrm{M} \gamma \frac{d x^{\mu}}{d t} \frac{d x^{\nu}}{d t}
$$

where $x^{\mu}=(c t, x)$ are the space-time coordinates along the kaon's world line, so a generalization of $(32)$ to this system is

$$
L_{\mathrm{int}}(\mathrm{K})=-\frac{1}{2} h_{\mu \nu} \bar{T}^{\mu \nu}
$$


where

$$
\bar{T}^{\mu \nu}=\left(\begin{array}{cc}
\alpha_{g} m & \Delta m / 2 \\
\Delta m / 2 & \bar{\alpha}_{g} m
\end{array}\right) \gamma \frac{d x^{\mu}}{d t} \frac{d x^{\nu}}{d t},
$$

and hence gravity can be introduced into Eq. (39) with the replacement [72]:

$$
S_{c l} \rightarrow S=S_{c l}+\int d t L_{\mathrm{int}}(\mathrm{K})
$$

The parameters $\alpha_{g}, \bar{\alpha}_{g}$ allow one possible violation of WEP, which corresponds to giving the $\mathrm{K}^{0}$ and $\overline{\mathrm{K}}^{0}$ gravitational accelerations of $\alpha_{g} g$ and $\bar{\alpha}_{g} g$, respectively, in a gravitational field where matter experiences an acceleration $g=|\nabla U|$. This model does not allow a violation of WEP in the off-diagonal elements of M, but this omission will not affect our results because the experimental data that we shall use relate to the diagonal elements alone.

For a horizontally directed kaon beam the potential $U$ can be regarded as constant because the change in gravitational potential energy during one $\mathrm{K}_{L}$-lifetime is very much smaller than the off-diagonal terms in the mass matrix M. Hence, the gravitational terms can be absorbed into re-definitions of the elements of the mass matrix, which then, apparently, become potential- and velocity-dependent. However, the influence of gravity on the units of length and time must be included before physical inferences can be drawn $[51,55,64]$. We shall assume that physical length and time measurements are made by instruments that obey WEP.

From Eq. (23) the effect of gravity on a time standard (clock) with Hamiltonian $H_{\text {clock }}$ is given by [51]

$$
H_{\text {clock }} \rightarrow H_{\text {clock }}+\frac{1}{2} h_{\mu \nu} \int T_{\text {clock }}^{\mu \nu}
$$

where we have assumed that the gravitational potential does not vary significantly over the dimensions of the clock. From (46) and von Laue's relativistic virial theorem [74]: $\int d^{3} x T_{i}^{i}=0$ for a closed system; we obtain $[51,55] H_{\text {clock }} \rightarrow\left(1+U / c^{2}\right) H_{\text {clock }}$ and so the clock frequency, based on transitions between different energy levels of $H_{\text {clock }}$, is redshifted [51]. Therefore, we may introduce local (physical) time units $\mathcal{T}=\left(1+U / c^{2}\right) t$ to replace the flat spacetime ones, $t[64]$.

Local length units can be obtained from the local time unit and the local speed of light, the influence of gravity on which is obtained [64] from the gravitationally modified action for the electromagnetic field (26). Treating the potential $U$ as constant over the propagation distances of interest, we find that the speed of light becomes $c \rightarrow c\left(1+2 U / c^{2}\right)$, and so local length units $\mathcal{L}=\left(1-U^{2} / c^{2}\right) L$ can be introduced to replace the flat spacetime ones, $L[64]$.

Using the physical time and length units, the kaon action in the presence of gravity can be written as

$$
S(t)=S_{c l}(t)-\int_{0}^{t} d t^{\prime}\left(\begin{array}{cc}
\left(\alpha_{g}-1\right) m & 0 \\
0 & \left(\bar{\alpha}_{g}-1\right) m
\end{array}\right) U \gamma\left(1+\frac{v^{2}}{c^{2}}\right),
$$

where $v$ is now the kaon's speed in physical units. In particular, note that when WEP is obeyed $\left(\alpha_{g}=\bar{\alpha}_{g}=1\right)$ physical quantities have their flat space-time values [64]. However, if the WEP is violated there would a $\mathrm{K}^{0}-\overline{\mathrm{K}}^{0}$ energy difference, which can equivalently 
be regarded as giving the $\mathrm{K}^{0}$ and $\overline{\mathrm{K}}^{0}$ effective (CPT-violating [51]) masses of

$$
m_{\mathrm{eff}}\left(\mathrm{K}^{0}\right)=m\left[1+\left(\alpha_{g}-1\right) \gamma^{2} \frac{U}{c^{2}}\left(1+\frac{v^{2}}{c^{2}}\right)\right]
$$

and

$$
m_{\mathrm{eff}}\left(\overline{\mathrm{K}}^{0}\right)=m\left[1+\left(\bar{\alpha}_{g}-1\right) \gamma^{2} \frac{U}{c^{2}}\left(1+\frac{v^{2}}{c^{2}}\right)\right]
$$

respectively, in the mass matrix [72]. (Note the large velocity-dependent enhancement of the tensor interaction.)

In the recent CERN experiment [13] that yielded the CPT test (3), the kaons had energies $\sim 100 \mathrm{GeV}$. Applying this constraint to the masses (48), (49), and using the potential of the supercluster, we obtain a constraint on the WEP violating parameters [72]:

$$
\left|\alpha_{g}-\bar{\alpha}_{g}\right| \leq 2.5 \times 10^{-18}
$$

Note that (50) does not demonstrate WEP for neutral kaons, because it only shows that gravity couples with essentially equal strength to the $\mathrm{K}^{0}$ and $\overline{\mathrm{K}}^{0}$ [64], not that the strength is the same as for ordinary matter [57]. However, from this result we can argue that gravity couples with essentially equal strength to quarks and antiquarks, and hence that the antiproton obeys WEP with the same precision as the proton [72].

Good's technique has also been applied to the results of electron-positron cyclotron frequency comparisons [75], but even with the future projected improvements in precision, the resulting WEP test for the positron would fall short of an antihydrogen null red-shift experiment by two orders of magnitude. But if the hydrogen and antihydrogen $1 S-2 S$ transition frequencies were found to be equal with a precision of one part in $10^{18}$, Good's method would imply equal gravitational couplings to the positron and electron with a higher precision of one part in $10^{14}$ (cf. Eq. (35), using the potential of the supercluster).

However, unlike the antihydrogen null red-shift experiment, Good's method assumes CPT-invariance, and so does not allow separate WEP and CPT tests - a constant frequency offset could be the result of either CPT or WEP violation. A conspiracy theory of cancelling CPT and WEP violations is highly improbable, but a null red-shift test of WEP for antiparticles is metrologically robust and hence preferable to Good's method for both positrons and antiprotons. For instance, Good's method requires the assumption that the gravitational inverse-square law holds over multi-Mpc (super- galactic) distance scales, whereas in a null red-shift experiment, the gravitational potentials involved are within the solar system, and so can be reliably calculated. (Although the range of the gravitational interaction is known to be at least several Mpcs, the inverse-square law has only been directly confirmed between laboratory and solar system distance scales [76].)

Good's argument illustrates one of the inescapable consequences of WEP- violation: physical quantities such as particle masses would become dependent on the local value of the gravitational potential [59], and hence on their location in the universe. (A similar potential dependence of physical quantities occurs if electric charge is not conserved [77]. Conversely, imposing potential-independence of physical quantities [47,67], as an additional requirement is equivalent to assuming WEP [78] if energy is conserved [59].) The potential-dependence of physical quantities could be compatible with the field equation satisfied by the potential if the graviton has a small mass, which would have to be less 
than that corresponding to the largest distance scale over which the inverse-square law has been tested. Departures from the inverse-square law on galactic distance scales have been proposed as an alternative to the introduction of dark matter for the explanation of galactic rotation curves $[79,80]$, but the massive graviton theory predicts a gravitational deflection of light that conflicts with observations [81]. Thus WEP-violation, if found, would require significant changes in the conventional view of the large-scale structure of the universe $[40]$.

We have shown above that antihydrogen is an extremely promising system for testing fundamental symmetries and measuring basic quantities in physics. We have also indicated that its experimental possibilities are greatly facilitated by its electrical neutrality and by the fact that it is composed of two stable antiparticles, one a heavy antiproton and the other a light positron. In spite of this, it must be noted that no antihydrogen atom has yet been knowingly produced. We must therefore turn our attention in the following sections to the practical problems of producing these constituents and persuading them to recombine.

\section{PRODUCING THE CONSTITUENTS OF ANTIHYDROGEN}

The characteristics of the atomic recombination reaction that produces antihydrogen, and the conditions under which high precision optical spectroscopy can be performed, effectively pre-determine the positron-antiproton initial conditions to be attained for efficient recombination to occur. The essential requirement here is that the relative velocities of the constituent particles be low enough to keep them within a few atomic radii of each other during the time required to radiate a photon or interact with another suitable third body. Moreover, if the produced atom moves off at high speed, either because of initial constituent motion or recombination-induced recoil, it will not be possible to perform high precision spectroscopic measurements on it. Thus the accumulation of significant numbers of cold antiprotons and positrons is a necessary first step in antihydrogen formation. Charged particles can be most easily cooled when they are stored in ion traps, so we must first consider the problems of trap loading with antiprotons and positrons.

\section{Antiprotons}

At present the world's most copious source of low-energy antiprotons is the PSLEAR (Proton Synchrotron - Low Energy Antiproton Ring) complex at CERN (Fig. 3) and most of the discussion which follows refer to this [82]. Protons accelerated to $26 \mathrm{GeV} / \mathrm{c}$ by the Proton Synchrotron strike a conversion target. Antiprotons of $3.5 \mathrm{GeV} / \mathrm{c}$ momentum, i.e. near the production maximum, are collected from the target and precooled in the Antiproton Collector ring and then are transferred to the Antiproton Accumulator where up to $10^{12} \overline{\mathrm{p}}$ can be stored for periods of weeks or even months. At regular intervals, from 15 min to several hours, batches of $10^{9}-10^{10}$ antiprotons are skimmed off from the stack and decelerated in the Proton Synchrotron from 3.5 GeV/c to $600 \mathrm{Mev} / \mathrm{c}$. This batch is then transferred to LEAR for further acceleration or deceleration and cooling. The antiproton energy is reduced in several steps, each step being followed by beam cooling (stochastic and/or electron cooling) to reduce the adiabatic phase-space increase which 
Figure 3: Schematic view of the the Proton Synchrotron — LEAR complex at CERN [82]. The details are explained in the text.

inevitably accompanies deceleration processes.

In its slow extraction regime (with spill lengths from $400 \mu$ s to about one hour) LEAR can deliver $3 \times 10^{9}$ antiprotons per filling at kinetic energy $5 \mathrm{MeV}$ or $1 \times 10^{9}$ antiprotons per filling at $2 \mathrm{MeV}$, whereas in fast extraction 50-400 ns long bunches of $>10^{8} \bar{p}$ are produced [82]. Ion traps can accept antiprotons of up to a few tens of $\mathrm{keV}$ for subsequent cooling ${ }^{1)}$. Thus the problem of decelerating antiprotons from $\mathrm{GeV}$ to $\mu \mathrm{eV}$ energies seems to be solved, except from the interval between a few $\mathrm{MeV}$ and a few tens of $\mathrm{keV}$.

\section{Antiproton deceleration}

Several schemes have been proposed for bridging this gap [83]. Here we shall summarise the three methods which have been experimentally tested at LEAR, namely degrading in a foil [84], the anticyclotron [85] and the RFQ decelerator [86].

- The degrading foil technique, in which a thin foil is used as the simplest of all possible decelerators, has been in successful use at LEAR for several years [84, 87, 88]. Behind its apparent simplicity lie several technical difficulties. These include the fabrication of flat surfaced, uniform thickness foils, since any departure from a perfect rectilinear foil geometry will add to the energy spread inevitably introduced by the statistical nature of the energy loss process. This latter characteristic strongly limits the efficiency of deceleration into a given energy interval in the beam leaving the foil. According to Monte-Carlo calculations, the theoretical efficiency limit for bringing $5.8 \mathrm{MeV} \overline{\mathrm{p}}$ to

1) Note that the concept of cooling means decreasing the momentum dispersion of a set of particles. For trapped particles, whose mean momentum should be about zero, this is equivalent to reducing their absolute kinetic energy. 
Figure 4: A schematic view of the technique to trap antiprotons using a foil moderator [84]. This method is used by the Harvard [87] and Los Alamos [66] groups at LEAR.

the $1-50 \mathrm{keV}$ range is $\epsilon \approx 6 \%$. The Harvard group [87] used a $300 \mu \mathrm{m}$ Be foil to slow down a fast-extracted LEAR bunch of $5.8 \mathrm{MeV} \bar{p}$ for subsequent capture in a Penning trap (see Fig. 4) as described in the next section. Using an $\mathrm{SF}_{6}$ gas cell combined with Mylar and Ti foils as a fine-tunable moderator, the same workers achieved $\epsilon(2-8$ $\mathrm{keV})=1.6 \times 10^{-4}$. The same method is used by the Los Alamos group [66], which is aiming to achieve a $1 \%$ efficiency with a much 'deeper' trap (see below).

- The Saclay group [86] has developed a radiofrequency quadrupole (RFQ) decelerator with a RF mass spectrometer which could be used to measure the charge-to-mass ratio of the $\bar{p}$. As the acceptance of the spectrometer at its injection energy, $200 \mathrm{keV}$, is orders of magnitude lower than the emittance of the $2 \mathrm{MeV}$ LEAR beam, the theoretical transmission efficiency limit is about $10^{-5}$. This RFQ cannot decelerate the $\bar{p}$ bunch sufficiently to fill a Penning trap of reasonable size, and its $\epsilon$ is too low in any case. A specially constructed RFQ might be envisaged and this option was considered by the Los Alamos group but rejected on the grounds of cost [66].

- The anticyclotron (Fig. 5) is a small superconducting cyclotron magnet without RF field, and was originally designed for studies on $\mathrm{X}$-ray cascades from antiprotonic atoms in low-pressure gases [89]. Deceleration is achieved by dissipating the particle energy in a dilute gas contained in what would be referred to in a normal cyclotron as the vacuum chamber. A low emittance $\bar{p}$ bunch is steered into the chamber through a thin entrance window lying in an axial plane at a radius of about $12 \mathrm{~cm}$. The continuously decelerating bunch then follows a spiral path. The resulting $\bar{p}$ swarm will form exotic atoms in the gas with a volume of a few $\mathrm{cm}^{3}$, near the magnet centre, if allowed to stop (the cyclotron trap configuration). However, it is possible to extract the bunch at a few $\mathrm{keV}$ kinetic energy through an axial bore-hole of the magnet using a high-voltage pulse or a suitably shaped magnetic field. This anticyclotron configuration was proposed to serve as a basic apparatus to provide an ultra-low energy antiproton beam [85] with a predicted transmission efficiency $\epsilon(7-10 \mathrm{keV})$ up to $20 \%$ with 0.3 mbar $\mathrm{H}_{2}$ as the moderator. Tests started at LEAR with $2 \mathrm{MeV}$ 
Figure 5: Scheme of the anticyclotron used as a gas target (cyclotron trap) for X-ray spectroscopy of muonic, pionic and antiprotonic atoms in low-pressure gases [89] and successfully tested for muon extraction at PSI [91].

antiprotons [90] using hydrogen and helium at a few mbar and continued at the Paul Scherrer Institute (PSI) with $4 \mathrm{MeV}$ negative muons and with a thin Mylar foil in the median plane as a moderating medium instead of a gas. In this configuration, a 5$25 \mathrm{keV} \mu^{-}$beam has been extracted from the anticyclotron with a $2 \%$ efficiency [91]. The foil moderator makes it possible to extract particles both in continuous and in pulsed regime, the former for atomic physics studies, the latter for experiments using Penning traps as a target volume or as a source of cold antiprotons. With LEAR antiprotons, at the predicted level of performance at $60 \mathrm{MeV} / c$, this efficiency might be as high as $25 \%$. According to the results of a computer simulation, the lower extraction efficiency expected at $105 \mathrm{MeV} / \mathrm{c}$ would be compensated by the higher number of particles from LEAR, giving roughly the same number of extracted antiprotons per unit time.

At present, the degrading foil technique seems to provide the best-understood and most economical solution to antiproton trap loading. The figure of $\epsilon(2-8 \mathrm{keV})=1.6 \times 10^{-4}$ quoted above is not much better than that of an RFQ decelerator and much poorer than that of the anticyclotron but higher efficiencies have recently been obtained by using deeper traps with larger angular acceptance [66, 94, 95]. Given the uncertainties in the rates of recombination reactions and in the number of $\bar{H}$ atoms necessary for high precision measurements, investigations into the technically more difficult but potentially more efficient anticyclotron method are being continued.

\section{Antiproton trapping and cooling}

Most of the methods under consideration for antihydrogen production proceed from the assumption that antiprotons will be available at thermal energies in a volume of order some $\mathrm{cm}^{3}$. The $\overline{\mathrm{p}}$ sample size will inevitably be many orders of magnitude smaller than it 
Figure 6: The main features of a Penning trap [92].

would be for the case of proton-electron recombination. The mean time for antihydrogen recombination to take place is correspondingly longer and the pressure in the system must be low enough to ensure that the antiprotons survive against annihilation on residual gas molecules much longer than this. In addition, annihilation of the antiprotons on the walls of the enclosure must of course also be avoided.

Such conditions were first met by a Harvard group with a configuration of electrostatic and magnetic fields known as a Penning trap [92] (Fig. 6. In this device, an axial magnetic field confines the charged particles' motion in the radial plane in cyclotron orbits, while a cylindrically symmetric system of electrodes results in a periodic motion along the z-axis. After passing a $5.8 \mathrm{MeV}$ fast-extracted LEAR bunch through a degrading foil as described above (Fig. 4), G. Gabrielse and his colleagues [88] were able to capture those antiprotons which emerged at less than $3 \mathrm{keV}$ energy in a $12 \mathrm{~cm}$ long trap of this form. The density of the residual gas in their trap was estimated to be less than 100 atoms $/ \mathrm{cm}^{3}$ (corresponding to a pressure of $\sim 10^{-17} \mathrm{mbar}$ ) from the life time of the antiprotons stored in the trap over a period of three months. Cooling the antiproton sample from $3 \mathrm{keV}$ to the temperature of the trap enclosure (4K) was also achieved by including electrons in the trap. These are able to dissipate the energy they receive in collisions with antiprotons much more rapidly via radiation than the antiprotons do themselves. Some tens of thousands of antiprotons were routinely trapped in this way. Experiments have also been done in which several $\bar{p}$ bunches were trapped in succession [87]. More than $10^{5}$ antiprotons were stacked in the $3 \mathrm{keV}$ trap in an hour, from 12 LEAR pulses. In optimal conditions, $10^{6}$ antiprotons might be trapped and cooled per hour. Other cooling methods are possible, including the coupling of the antiprotons' motion to a dissipative external resonant circuit [93], but the electron method is the only one which has been extensively 
tested with antiprotons at the time of writing.

M. H. Holzscheiter et al. (Los Alamos) have recently been continuing along the above lines, and aim to increase the trapping efficiency to about $2.5 \%$. This is mainly to be achieved by using a larger and deeper trap designed to catch antiprotons emerging from a degrading foil with energies between $1 \mathrm{keV}$ and $30 \mathrm{keV}$ [66]. It might be possible to double this efficiency if suitable high voltage switches can be developed which allow the trap to be operated at its design potential of $60 \mathrm{kV}$. In tests done during July and August 1992, approximately $3 \times 10^{4}$ antiprotons were trapped in this system [94] and in 1993 this number was increased to about one million [95]. Although uncertainties in the beam conditions do not permit an absolute efficiency estimate, time of flight spectra taken with the trapping electrodes switched off indicate that the number of antiprotons emerging from the foil in the energy range corresponding to the trap depth are quite consistent with the calculations used in designing the trap. Taking these figures, it should be possible to trap $2.5 \times 10^{6}$ particles per single LEAR bunch of $10^{8}$ antiprotons. If successive bunches can be stacked as the Harvard group has demonstrated [87], only four LEAR spills of $10^{9}$ $\bar{p}$ each would be needed to accumulate $10^{8}$ cold antiprotons over a period of a few hours.

It does not then seem unreasonable that LEAR will soon be able to produce $10^{7}-10^{8}$ cold trapped antiprotons per hour (roughly $10^{8}-10^{9}$ per day). With the anticyclotron at full efficiency this might be an order of magnitude higher still.

\section{Positrons}

\section{Positron production}

The efficient production of low energy positrons has been the subject of detailed scrutiny over the last two decades and most of the basic physics is understood and the relevant technical problems solved (see, e.g., [96, 97]). Thus it should be feasible to construct a device in the LEAR experimental hall to produce, trap, and perhaps cool $\mathrm{e}^{+}$in conjunction with a trap containing antiprotons [88].

The basic mechanism behind the production of low energy $\mathrm{e}^{+}$is known as moderation and involves the slowing down of initially high energy $(\approx \mathrm{MeV})$ positrons in a solid material followed by their diffusion to a surface where they may be emitted into vacuum with a kinetic energy of the order of $1 \mathrm{eV}$. This can occur if the surface has a positron work function $\left(\Phi_{+}\right)$which is negative, something not uncommon for metals where the surface dipole barrier is favourable (i.e. it enhances instead of hindering positron evaporation [96]), or if the $\mathrm{e}^{+}$arrive at the surface with sufficient kinetic energy to overcome a positive work function. The slow $\mathrm{e}^{+}$thus produced can be further manipulated by accelerating or cooling if desired. The main inefficiency in $\mathrm{e}^{+}$moderation arises from the annihilation-limited $\mathrm{e}^{+}$diffusion length $\left(10^{2}-10^{3} \mathrm{~nm}\right)$ in solids, which is much shorter than the mean implantation depth of the high-energy $\mathrm{e}^{+}$. Efficiencies of fast-slow conversion therefore depend greatly upon the positron kinetic energy and vary from $10^{-2}-10^{-4}$ for positrons from a radioactive source, to $10^{-6}$ for pair-produced fast positrons from a $100 \mathrm{MeV}$ electron accelerator (see, e.g. [97, 99]). The production of low energy positrons and positronium in the cryogenic environment common in particle traps also appears feasible [100, 101], although this issue is currently controversial for the situation where clean surfaces must be prepared and maintained in ultra-high vacuum conditions [104].

One method to manipulate slow $\mathrm{e}^{+}$is to construct a beamline (see, e.g., $\left.[96,105]\right)$. 
Figure 7: Schematic plan view of the magnetically transported positron beam in use at University College London [97]. The apparatus is approximately $3.5 \mathrm{~m}$ in total length. The source and the deflector end are electrically isolated from the remainder of the beamline. The moderator can be remotely withdrawn into the side chamber for annealing

A representative example, as shown in Fig. 7, is in use at University College London and delivers a continuous beam of $10^{5} \mathrm{e}^{+} / \mathrm{s}$ which is variable over a wide energy range [97]. The positrons are derived from a commercially available ${ }^{22} \mathrm{Na}$ source held in close proximity to a moderator (typically a single crystal foil or tungsten mesh). Low energy $\mathrm{e}^{+}$are confined by an axial magnetic field of around $10^{-2} \mathrm{~T}$ produced by a series of coils placed in the Helmholtz configuration. They are accelerated to $100 \mathrm{eV}$ and deflected by $25 \mathrm{~mm}$ using an $E \times B$ filter so that the remainder of the beamline is not in the line of sight of the radioactive source. The final beam energy is fixed by the potential difference which can be placed between the isolated source-deflector region and the remainder of the beamline. The latter is, in the instrument shown, to be mainly applied to atomic scattering studies with positrons and positronium. The details of the apparatus are not shown in Fig. 7 as they are not relevant to the present discussion, however, $\mathrm{e}^{+}$beams of this type have been used for a wide variety of studies in surface and atomic physics and as input to a positron trap (see below).

At some laboratories pulsed, low-energy positron beams are formed by using pair production at electron accelerators and it is worth examining, in the light of the possibilities available at CERN (see below), the types of source/moderator configurations employed at these facilities. Two examples are shown in Figs. 8a and 8b. The former shows the arrangement of Howell et al. [106] used to produce pulsed $\mathrm{e}^{+}$at the Lawrence Livermore $120 \mathrm{MeV}$ linear accelerator. The maximum measured efficiency of this arrangement is a fast $\mathrm{e}^{-}-$slow $\mathrm{e}^{+}$conversion of $1.5 \times 10^{-6}$. Figure $8 \mathrm{~b}$ shows the target - moderator system of Mohri et al. [107] which has been fully modelled using the EGS-4 (Electron Gamma Shower) simulation code [108] and has a projected yield of $7 \times 10^{-5}$ slow $\mathrm{e}^{-}$per incident $35 \mathrm{MeV}$ electron. The main differences between this modular photon - positron 
converter and the more traditional target/converter (Fig. 8a) are the stacking of tungsten foils in which the bremsstrahlung photons are converted directly to $\mathrm{e}^{+}$, and the efficient extraction of the slow $\mathrm{e}^{+}$which are emitted from the large surface area. Thus it would appear that the medium energy, high current electron accelerator can provide very intense $\mathrm{e}^{+}$pulses. The performance of this system is currently being evaluated at Kyoto and preliminary results are encouraging [109].

Once slow $\mathrm{e}^{+}$have been produced either in continuous or pulsed form they can be manipulated in many ways. Of particular importance is the remoderation/brightness enhancement procedure [110] whereby the positrons are accelerated to a desired energy and focussed (usually by means of electromagnetic lenses) onto a target from which they can be re-emitted with lower energies but from a spot size characteristic of the primary beam. This process can be efficient $(\approx 30 \%[111])$ since the primary $\mathrm{e}^{+}$kinetic energy is so low that the implantation depth and diffusion length are of the same order. In addition, low energy $\mathrm{e}^{+}$can be used to form positronium in vacuum in a number of ways.

\section{Positronium production}

The collision of $\mathrm{eV}-\mathrm{keV}$ positrons with a suitable surface can produce positronium (Ps) atoms $[101,112]$ with high efficiency. When $7 \mathrm{keV} \mathrm{e}{ }^{+}$were implanted in $\mathrm{SiO}_{2}$ powder, $57 \%$ of them formed Ps [101], whereas in transmission geometry through thin $\operatorname{Ag}(100)^{2)}$ foils at $800 \mathrm{~K}$, a conversion efficiency of incident positrons to Ps of $12 \%$ was obtained [112]. The wide variety of techniques which can be used for such purposes have been reviewed in $[96]$.

Figure 9 presents Ps energy spectra in vacuum measured perpendicular to a clean Al(111) surface held at temperatures of $303 \mathrm{~K}$ and $692 \mathrm{~K}$ following bombardment by $2 \mathrm{keV}$ positrons [98]. It can be seen that when $E_{\perp}$ (the energy perpendicular to the surface) is high, these spectra overlap. The process responsible for this temperature independent phenomenon is the so-called prompt emission of $\mathrm{e}^{+}$as Ps following thermalisation of the positrons in the bulk and their diffusion to a surface. The maximum energy of emission is the formation potential $\epsilon_{\mathrm{Ps}}=\Phi_{+}+\Phi_{-}-6.8 / n^{2}$ where $\Phi_{-}$is the electron work function of the surface and $6.8 / n^{2}$ is the binding energy, in $\mathrm{eV}$, of the Ps in a state with principal quantum number $n$. For $n=1 \epsilon_{\mathrm{Ps}}$ is usually negative for metals since $\Phi_{+}$is often negative or slightly positive.

By contrast, the Ps yield at the lower $E_{\perp}$ is greatly enhanced as the temperature of the sample is raised. This is due to thermal desorption of surface trapped $\mathrm{e}^{+}$and provides a useful source of very low energy positronium. The activation energy, $E_{a}$, of the desorption process is given by $E_{a}=E_{b}+\Phi_{-}-6.8 / n^{2}$ where $E_{b}$ is the binding energy of the positron at the surface.

In many experiments it may be necessary to separate, both physically and electrically, the $\mathrm{e}^{+}$beam and the Ps. This can be achieved by injecting positrons at an appropriate energy on one side of a thin foil, low energy Ps being emitted from the other side. Ps formation in this transmission geometry has been demonstrated [112] using a $190 \mathrm{~nm}$ thick $\operatorname{Ag}(100)$ foil which has the added virtue of having $\Phi_{+}>0$. The results of this study are presented in Fig. 10 which shows the relative Ps fraction thermally desorbed from the foil when held at a temperature $800 \mathrm{~K}$.

\footnotetext{
2) The digits in brackets are the Miller indices indicating the crystal orientation of the surface
} 
Figure 8: (a) Target and moderator assembly used to produce low energy positrons at the Livermore electron linac. (b) Modular photon - positron converter for very intense, slow positron fluxes; this device is under test at Kyoto. 
Figure 9: Energy spectra of positronium emitted from an A1(111) surface heated to two different temperatures [98]. The thermally activated component is clearly seen in the data at $692 \mathrm{~K}$. 
Figure 10: The energy dependence of the conversion efficiency of $\mathrm{e}^{+}$into thermally activated $\mathrm{Ps}$ in transmission from a thin silver foil relative to the backscattering yield at $100 \mathrm{eV}$ impact [112].

In both of the above cases the lowest kinetic energy Ps were produced by desorption from a heated sample. In foreseeable $\overline{\mathrm{H}}$ formation scenarios it may be desirable to have a Ps formation/emission mechanism operable at cryogenic temperatures. Ps can be formed by $\mathrm{e}^{+}$slowing down in the bulk of many insulating materials [96] and can be emitted from the surface if the work function is negative. The first demonstrations of this phenomenon [113, $114,115]$ were for fast $\mathrm{e}^{+}$stopping in powdered materials and more recently, Mills and $\mathrm{co}^{-}$ workers [101] have studied $\mathrm{Ps}$ emission from $\mathrm{SiO}_{2}$ powders held at different temperatures following low-energy $\mathrm{e}^{+}$implantation. In this experiment a time-of-flight technique was used to estimate the energy spectrum of the Ps that diffused out of the powder sample and into the surrounding vacuum chamber. Figure 11 shows spectra obtained at 7, 10, and $19 \mathrm{keV}$, respectively. Note, in particular, the progressive reduction in the average kinetic energy as the temperature of the powder was lowered. Such a target has obvious cryogenic applications.

The formation of antihydrogen in collisions between antiprotons and Ps produced in vacuo is discussed in some detail below. As the formation cross-section is strongly dependent on the Ps principal quantum number $n$, the reaction will proceed more readily if the Ps is in a Rydberg, or highly excited state. We note that such Ps states $(n=13-19)$ have been produced by two-photon excitation using the pulsed $\mathrm{e}^{+}$beam at Lawrence Livermore Laboratory [116]. 
Figure 11: Energy spectra of $\mathrm{Ps}$ emitted from a $\mathrm{SiO}_{2}$ powder sample at three different temperatures [101]. The dashed lines correspond to the relevant Maxwellian distribution. 


\section{Positron trapping, cooling, and bunching}

Most of the methods used so far have employed low energy $\mathrm{e}^{+}$beams as a starting point, with radial confinement provided by a magnetic field. The basic idea (which is the same whether the positrons come from a continuous or a pulsed source) is to load a region in which the $\mathrm{e}^{+}$are trapped before ejecting them in a bunch as required by the particular experiment.

At a pulsed source, trapping is straightforward because the arrival time of the $\mathrm{e}^{+}$ burst at the trap entrance is known. As an example, the formation of a high density $\mathrm{e}^{+}$ plasma $\left(\approx 10^{10} \mathrm{~cm}^{-3}\right.$ in a $1 \mathrm{~mm}$ diameter, $30 \mathrm{~cm}$ long column) at low temperatures is being pursued at the Livermore machine [117].

Several schemes have been devised to trap and accumulate positrons from a lowenergy, continuous beam. One of these consists of a Penning-type trap arrangement in which the $\mathrm{e}^{+}$are injected over a voltage barrier but then lose energy in the trap by collisions with deliberately introduced gas molecules [118]. To date a cloud containing around $10^{7} \mathrm{e}^{+}$has been confined in a region of order $1 \mathrm{~cm}$ in size and had a lifetime of $40 \mathrm{~s}$ [119]. The latter is determined solely by the presence of the moderating gas $\left(\approx 10^{-6}\right.$ torr of $\left.\mathrm{N}_{2}\right)$ which also means that this instrument cannot be directly interfaced to an antiproton trap where extremely high vacuum is necessary for long $\bar{p}$ lifetimes [88].

A second device has the moderator serving as one of the trapping electrodes, its potential being ramped upwards as a function of time to prevent the returning $\mathrm{e}^{+}$from striking it [120]. This apparatus is based on the $\vec{E} \times \vec{B} /|\vec{B}|^{2}$ drift of the orbit of the positron introduced by the non-uniform electrostatic fields (superimposed on a uniform axial magnetic field) used to reflect the $\mathrm{e}^{+}$. More details on the performance of this device, including the importance of the moderator geometry, can be found in [120]. Although this trap does not rely on gas moderation, its applicability may still be limited to low magnetic fields which will result in a large enough cross field drift. Typical magnetic fields used in $\bar{p}$ traps are nearly 100 times greater than that at which this trap has been operated [120].

A third scheme, due to Mills [121], is illustrated in Fig. 12 and shows how a trap and ejection buncher can operate. The trap, Fig. 12a, uses a combination of an electrostatic and a magnetic mirror. The former is achieved by the voltage applied to the grid $G 1$ which the injected $\mathrm{e}^{+}$have insufficient axial kinetic energy to overcome, whilst the latter is formed by the high magnetic field $\vec{B}_{m}$ which is approximately twice $\vec{B}_{0}$, the uniform field elsewhere. The positrons which enter over the magnetic mirror have their cyclotron motion excited by the tuned radiofrequency transverse electric field, which ensures reflection by the magnetic mirror on return from $G 1$.

Fig. $12 \mathrm{~b}$ shows that $G 1$ is part of a larger series of electrical elements which form the buncher. Typical distances are $\approx 1 \mathrm{~m}$ with around a hundred individual elements forming an accelerator region capable of producing $\mathrm{e}^{+}$bursts at the target $T$. This is achieved by suddenly applying a potential which varies quadratically with the distance $z$ from $T$ and it is easy to show that, ideally, the $\mathrm{e}^{+}$inside the buncher then arrive simultaneously at $T$. There are a number of ways to apply this voltage to overcome some of the effects which lead to a broadening of the arrival times. In the solution shown in Fig. 12b, the latter half of the buncher is d.c. biased, including $G 1$, such that only the first half is used as a trap. This helps to reduce the effect of time straggling of $\mathrm{e}^{+}$which would have been ejected from the second half of the buncher with relatively low energies $\left(\leq e V_{0}\right)$. Ejection 
Figure 12: (a) Combined magnetic bottle and electrostatic trap to load positrons in the buncher prior to ejection [121]. Here $\vec{E}$ is the transverse, radiofrequency, electric field to excite the $\mathrm{e}^{+}$cyclotron motion. The square objects represent the coil windings. All other symbols are defined in the text. (b) Schematic illustration of the harmonic potential buncher developed by Mills [121]. The direction of the axial magnetic field $\vec{B}$ is shown together with the positron moderator $S$. The voltages $V_{S}$ and $V_{S}^{\prime}$ are nearly the same to optimise trapping with a small potential, of the order of the input beam energy (here $<1 \mathrm{eV}$ ). 
Figure 13: Penning trap for continuous trapping of positrons developed by Haarsma et al. [122]. The ${ }^{22} \mathrm{Na}$ source (not shown) is located around $25 \mathrm{~cm}$ above the centre of the trap. The magnetic field strength at the source is $1.7 \mathrm{~T}$ and at the trap $5.9 \mathrm{~T}$.

from the first part of the buncher is achieved by suddenly applying a voltage $3 V_{0}$ to the grid $G 2$. A device of this type has been reported as being $\approx 50 \%$ efficient and produces positron bursts of $\approx 10 \mathrm{~ns}$ width at a repetition rate of around $10 \mathrm{kHz}$ [102]. This type of instrument has not yet been used to accumulate large quantities of positrons.

One drawback of this technique is the use of a radiofrequency field to affect trapping by excitation of the positron cyclotron motion. Whilst this presents no problems for the magnetic fields used in most low-energy positron beams (radiofrequency of the order of $500 \mathrm{MHz}$ ) the frequency required in a $6 \mathrm{~T}$ field is $168 \mathrm{GHz}$, which may be impractical. This type of instrument, perhaps in common with the others described above, could be used to inject positron bursts into a trap with a high magnetic field to allow the interaction of $\mathrm{e}^{+}$or Ps with stored antiprotons. In addition to these devices there are, however, other schemes, based to some extent on the work of Dehmelt and coworkers [24], wherein low energy $\mathrm{e}^{+}$can be trapped without using a beamline but still employing moderator technology. In this scheme $\mathrm{e}^{+}$are injected off the symmetry axis of a Penning trap so that their subsequent magnetron motion allows them to be trapped and rapidly cooled. Currently, two such schemes are being investigated [122, 123]. In principle, this method offers continuous loading and cooling, and may provide high positron densities, perhaps up to $10^{9} \mathrm{~cm}^{-3}$ at cryogenic temperatures, because the $\mathrm{e}^{+}$lifetime in the trap is expected to be long.

Figure 7 shows the trap system under development by Haarsma et al. [122]. Here high-energy positrons from a ${ }^{22} \mathrm{Na}$ source (not shown) are guided down the fringe fields produced by a $6 \mathrm{~T}$ solenoid, through small apertures which form part of the eventual trap, 
and onto a tungsten moderator. Slow $\mathrm{e}^{+}$thus produced are guided by the magnetic field back into the trap though - as noted above - off its symmetry axis, where they may be trapped if they have insufficient energy to overcome the electrostatic barrier at the edge of the trap. Very recently this instrument has been reported [103] as having trapped in excess of $10^{4} \mathrm{e}^{+}$at a temperature of $4.2 \mathrm{~K}$.

Turning now from general possibilities to the more restrictive conditions near an antiproton source, we briefly consider the following options for sources of high-energy positrons in the context of LEAR:

1. a strong radioactive source (several hundred $\mathrm{mCi}$ );

2. an existing high-energy electron beam transported into the antiproton area to form slow $\mathrm{e}^{+}$by the bremsstrahlung - pair production - moderation scheme;

3. a dedicated high-current medium-energy electron accelerator.

Option (1) appears to be the simplest, particularly if a trap can be loaded continuously with $\mathrm{e}^{+}$without the use of a slow $\mathrm{e}^{+}$beamline. Option (2) is, unfortunately, rather unattractive. Estimates made by the LEAR machine group [124] show that only about $10^{10}$ electrons per sec at $600 \mathrm{MeV}$ could be delivered to the antiproton area and this will yield around $10^{5} \mathrm{e}^{+} / \mathrm{s}$. The level of radiation produced in the electron target region (see Fig. 8) demands that a slow $\mathrm{e}^{+}$beamline be constructed, although the slow $\mathrm{e}^{+}$intensity would probably be less than that obtained if a radioactive source was used.

Option (3) may, in the long term, be the most desirable if antihydrogen is to be produced on a routine basis for study by a number of different groups. A small dedicated machine, perhaps a microtron, could be used in conjunction with a slow positron beamline to produce a pulsed beam of more than $10^{7} \mathrm{e}^{+} / \mathrm{s}$.

\section{MAKING THE ANTIHYDROGEN}

\section{Antihydrogen formation}

Several schemes have been proposed for antihydrogen production. Not all of them are suitable for experiments at the highest level of precision discussed in the introductory and spectroscopic sections of this review but may nevertheless play an important role in the initial development of antihydrogen physics. We therefore include in this section a discussion of all methods so far suggested for the synthesis of antihydrogen from its constituents.

Antihydrogen production by radiative recombination

The first proposal for antihydrogen formation was based on the spontaneous radiative recombination reaction

$$
\overline{\mathrm{p}}+\mathrm{e}^{+} \rightarrow \overline{\mathrm{H}}+h \nu,
$$

between antiproton and positron beams [125] and was subsequently developed by Herr et al. [126]. The cross-section for the matter-conjugate reaction,

$$
\mathrm{p}+\mathrm{e}^{-} \rightarrow \mathrm{H}+h \nu
$$

has been calculated by many authors [127]-[136]. For capture into the $n=1$ level the 
Figure 14: Laser-enhanced radiative recombination of electrons and protons at the Trondheim Storage Ring [156]. $Q, D$ and $C$ denote quadrupole, dipole and correction magnets. The electron beam and the circulating proton beam are merged in the magnetic field of the electron cooler. The laser beam is adjusted to travel antiparallel to the beam of spontaneously formed hydrogen atoms and reflected by a thin quartz window, through which the recombined hydrogen atoms pass before detection.

cross-section is inversely proportional to the centre-of-mass energy, $E$, of the system:

$$
\sigma=\frac{2^{8} \pi^{2}}{3 \mathrm{e}} \alpha^{3} a_{0}^{2} \frac{I_{0}}{E}
$$

for $E \ll I_{0}=\alpha^{2} m c^{2} / 2$ the ionization energy [137] ( $\mathrm{e}=2.718 \cdots$ and $a_{0}$ is the Bohrradius). Note that capture into higher- $n$ levels is suppressed by a factor of $n^{-3}$. Hence, the reaction rate will be maximized with co-moving particles. However, because of the proportionality of the cross-section for such radiative processes to the third power of the fine-structure constant, very low production rates of only a few atoms per second could be expected at LEAR. For this reason, laser-stimulated recombination

$$
\overline{\mathrm{p}}+\mathrm{e}^{+}+n h \nu \rightarrow \overline{\mathrm{H}}+h \nu+(n+1) h \nu .
$$

was later proposed $[138,156]$ in which the rate could be increased by about two orders of magnitude for capture into particular $n$-states, by illuminating the reaction region with photons with an energy $h \nu$ corresponding to that of the emitted photon in the spontaneous reaction (Fig. 14). However, with existing laser technology these large gain factors can only be achieved for $n>8$, for which the spontaneous cross-section is already suppressed by a large factor of $n^{-3}$ as compared to the $n=1$ capture and so the overall enhancement of the rate is typically small.

For the hydrogen case, radiative recombination has already been achieved with merged proton and electron beams in a storage ring $[157,158]$. In one of these tests, a $330 \mathrm{keV}, 10 \mathrm{nA}$ proton beam was merged over a length of $86 \mathrm{~mm}$ with a $180\left( \pm 5 \times 10^{-4}\right) \mathrm{eV}$, $100 \mu \mathrm{A}$ electron beam (both energies correspond to $\beta=0.0265$ ) [158] (Fig. 14). A small section of the interaction volume was illuminated by a $20 \mathrm{~W} \quad \mathrm{CO}_{2}$ laser and an increase in the reaction rate of more than an order of magnitude was obtained in the transition to the $n=12$ hydrogen level. Illumination of the full interaction volume would have resulted in a rate more than three orders of magnitude above that for spontaneous recombination. Rate estimates for the $\overline{\mathrm{H}}$ case, assuming typical LEAR conditions for circulating $\overline{\mathrm{p}}$ and $7 \times 10^{7}$ positrons $/ \mathrm{cm}^{3}$, range from $10 \overline{\mathrm{H}} / \mathrm{s}[159]$ to $10^{4} \overline{\mathrm{H}} / \mathrm{s}[160]$. 
As well as having very low rates, the in-beam radiative reactions yield neutral antihydrogen atoms that are moving off at very high velocities, which makes it extremely difficult to perform any subsequent experiment on them. However, in 1986 Gabrielse et al. proposed [139] that the low rate could be overcome to some extent by using cryogenic, trapped antiprotons and positrons, because the long confinement times would allow many antiproton-positron encounters to take place with low relative velocities. Moreover, the produced antihydrogen atoms would also be moving at low speed. The spontaneous production rate (ignoring the electromagnetic fields in the trap) per trapped antiproton with a positron density of $n_{e}\left[\mathrm{~cm}^{-3}\right]$ in an antiproton-positron plasma at a temperature $T$ $\left(k T \ll I_{0}\right)$ is $[137]$

$$
\Gamma=35 \alpha^{3} a_{0}^{3} n_{e} \frac{I_{0}}{\hbar}\left(\frac{I_{0}}{k T}\right)^{\frac{1}{2}}
$$

giving $[60]$

$$
\Gamma=3 \times 10^{-11}\left(\frac{4.2}{T}\right)^{\frac{1}{2}} n_{e}\left[\mathrm{~s}^{-1}\right]
$$

which shows a $T^{-1 / 2}$ rate enhancement with decreasing temperature. The trap's magnetic field only affects the cross-section for recombination into high- $n$ states which makes small contribution to the rate [136]. However, assuming a positron density of $n_{e}=10^{7}$ $\mathrm{cm}^{-3}$ and $10^{4}$ trapped antiprotons, this gives a production rate that is still only a few atoms per second at a temperature of $4.2 \mathrm{~K}$ [60]. Laser-stimulated recombination inside a trap would have an equally low rate. Thus, attention has turned to two other in-trap recombination reactions with intrinsically higher cross-sections: antiproton-positronium charge exchange [140],

$$
\overline{\mathrm{p}}+\mathrm{Ps} \rightarrow \overline{\mathrm{H}}+\mathrm{e}^{-}
$$

and the three-body recombination reaction

$$
\overline{\mathrm{p}}+\mathrm{e}^{+}+\mathrm{e}^{+} \rightarrow \overline{\mathrm{H}}+\mathrm{e}^{+}
$$

in which a spectator particle carries away the excess energy and momentum released in the recombination [165]. We shall examine these two new reactions in turn.

\section{Antihydrogen production in collisions of antiprotons with positronium}

The advantage of this antihydrogen production reaction is its large calculated crosssection (of order $10^{-15} \mathrm{~cm}^{2}$ ) $[161,162]$. It has been pointed out $[163,164]$ that even this large cross-section can be enhanced by several orders of magnitude if the positronium can be prepared in a highly excited state. The radius of the positronium atom depends on the square of the principal quantum number $n$, so that the capture cross-section should scale as $n^{4}$. The procedures involved in producing such Rydberg states of the positronium atom have been discussed in detail elsewhere [116, 163]. Essentially, an excimer laser simultaneously pumps two dye lasers which induce first the $1 S-2 P$ transition $(5.1 \mathrm{eV})$ and then the transition from $n=2$ to the required Rydberg state $(\sim 1.7 \mathrm{eV})$. Another good feature of the process is that the resonant capture condition, $n_{\mathrm{H}} \sim \sqrt{2} n_{\mathrm{Ps}}$ favours production in relatively low- $n$ states.

The proposed experimental setup is the following [3, 163]: Positrons from a ${ }^{22} \mathrm{Na}$ source of some $500 \mathrm{mCi}$ are moderated and electrostatically focussed in transmission 
Figure 15: Laser-enhanced antihydrogen formation on excited positronium [163]. The Ps atoms, formed at the trap walls in ground state, are excited by a laser pulse and form an excited $\overline{\mathrm{H}}$ atom on an antiproton stored in the Penning trap

or backscattering geometry onto a Ps converter, which is a thin foil with an oxide or condensed gas layer on it (Fig. 15). As summarised before [97] the converter can be prepared to emit slow Ps atoms in the low temperature environment typical of that found in ion traps. Here they can be excited as described in [116] by suitable combinations of lasers to states in the neighbourhood of $n=10-20$. When the antihydrogen-forming reaction takes place with a Rydberg state of positronium, the resulting $\overline{\mathrm{H}}$ atoms should be preferentially formed in states with wave functions which best overlap that of the Ps atom, and would thus have a binding energy close to that of Ps (taking the case of $n=10$ Ps, the antihydrogen states would be distributed around $n=7-13$ [163]). They would then be confined in a neutral particle trap as discussed below, where they would rapidly deexcite to the ground state, which as we have seen is the most interesting for testing fundamental principles and symmetries.

With $10^{7}$ cold $\bar{p}$ from one LEAR fill, and a primary positron beam of $10^{7} \mathrm{~Hz}$ striking the Ps converter, a yield of $10-100 \overline{\mathrm{H}} / \mathrm{s}$ can be envisaged. Allowing for the depletion of the $\bar{p}$ stack, the method can be expected to produce $2 \times 10^{6}$ cold antihydrogen atoms per day $[3,162]$ for ground state spectroscopic studies as described in Section 4. Work is under way in Aarhus to simulate this process for the case of proton-electron recombination to hydrogen atoms.

Antihydrogen production in $\overline{\mathrm{p}}+\mathrm{e}^{+}+\mathrm{e}^{+}$collisions

At first sight it appears unlikely that this reaction would have a larger cross-section 
than spontaneous radiative recombination [141]. However, several authors have calculated the corresponding cross-section with matter,

$$
\mathrm{p}+\mathrm{e}^{-}+\mathrm{e}^{-} \rightarrow \mathrm{H}+\mathrm{e}^{-},
$$

for astrophysical and plasma physics purposes [142]-[146] and simple dimensional arguments [136] indicate that the antihydrogen formation rate for trapped antiprotons and positrons via this reaction is

$$
\Gamma=6 \times 10^{-12}\left(\frac{4.2}{T}\right)^{9 / 2} n_{e}^{2}\left[s^{-1}\right]
$$

per trapped antiproton [165] which can be much larger than the spontaneous radiative recombination rate at low temperatures.

A potential drawback of this reaction is that from elementary energy conservation arguments, the atoms will be formed in highly excited $(n \sim 200)$ Rydberg $(l \sim n-1)$ states, which are not only weakly bound, and hence susceptible to collisional re-ionization, but also have long radiative lifetimes $\left(\sim n^{3} l^{3}\right.$ times typical allowed dipole lifetimes, i.e. minutes [147]). Thus collisional deexcitation is more effective but can only proceed down to $n \sim 40$ at which point radiative transitions will dominate. These considerations, however, have ignored the role of the trap's magnetic field. Its importance may be gauged from the fact that the cyclotron radius for a positron in a $6 \mathrm{~T}$ magnetic field at $4 \mathrm{~K}$ is

$$
\rho_{c}=\frac{\sqrt{2 k T m}}{e B} \sim 3 \times 10^{-7}[\mathrm{~m}]
$$

whereas the radius of an antihydrogen atom in an $n \sim 200$ Rydberg state is

$$
n^{2} a_{0} \sim 2 \times 10^{-6}[\mathrm{~m}]
$$

The influence of the small cyclotron radius on the ternary recombination rate has been calculated by Glinsky and O'Neil [148] who found a reduction by an order of magnitude. However, this treatment does not take into account the effect of the magnetic field on the atomic states. Indeed, for $n>26$ in a $6 \mathrm{~T}$ field the spacing of the positron Landau levels $\left(\mu_{B} B\right)$ is greater than the spacing of the adjacent Coulomb states $\left(13.6 \mathrm{eV} / \mathrm{n}^{3}\right)$, which shows that $n$ is not a good quantum number under these conditions. The behaviour of a highly excited (anti)hydrogen atom in very strong magnetic fields [149] is still an unsolved problem that exhibits chaotic features [150]. Thus, a complete analysis of ternary recombination in an ion trap is a complex problem that awaits a full solution.

Technically, this method requires the antiprotons and the positrons to be confined simultaneously in a small volume. This can be achieved by a nested pair of opposite-sign potential wells within a superimposed magnetic field [60, 165] (Fig. 16). The potential barrier between the two traps would be lowered to allow the antiprotons to pass repeatedly through the positron cloud when the antiprotons are sufficiently cold and compressed. With positrons from a radioactive source - moderator arrangement continuously accumulated and cooled to $T=4.2 \mathrm{~K}$ at densities of $10^{7} \mathrm{~cm}^{-3}$, this method should yield [165] about $600 \overline{\mathrm{H}} / \mathrm{s} /($ trapped $\overline{\mathrm{p}})$. Most of the contents of the trap, $10^{6} \overline{\mathrm{p}}$, would be converted into antihydrogen in an hour (corresponding to an antihydrogen yield of $2 \times 10^{7} /$ day). 
Figure 16: Antihydrogen formation in nested antiproton and positron traps [166]. The cylindrical electrodes $E n$ provide the antiproton reservoir $(E 1-E 5)$ and the nested trap $(E 5-E 9)$ for recombination. The axial potential distribution, $V(z)$, is also shown.

As is the case for the positronium reaction, testing the method by the corresponding process in which electrons and protons recombine to hydrogen is difficult since there is no unambiguous annihilation signal to indicate the presence of hydrogen atoms. In preparatory experiments Quint et al. [166] have passed swarms of positive and negative particle species from neighbouring traps (electrons and protons or positive helium ions) through each other. The trapped charged particles were counted by ejecting them onto microchannel plates. Mindful that antiproton samples for antihydrogen production may have to be removed to locations far from the noisy electromagnetic environment typical of particle accelerators, members of the same group [167] have transported electrons in a superconducting Penning trap at $5 \times 10^{-17}$ torr over a distance of some 5000 kilometres.

In summary, the great advantage of this method is the expected high rate; its principal disadvantage the slowness and possible inefficiency of spontaneous deexcitation of the antihydrogen Rydberg atom to the more interesting low $n$ states. This latter problem can perhaps be solved by radiative stimulation of the deexcitation process.

\section{Antihydrogen production at $\mathrm{GeV}$ energies}

A recent proposal considers $\overline{\mathrm{H}}$ production at $\mathrm{GeV}$ energies in the $\mathrm{E} 760$ experiment, which studies $\mathrm{p}-\overline{\mathrm{p}}$ annihilation at the Fermilab $\overline{\mathrm{p}}$ accumulator using an internal gas jet target. The authors [168] estimate the fraction of $\mathrm{e}^{-}-\mathrm{e}^{+}$pairs produced in this target by the circulating $3 \mathrm{GeV} / c$ antiproton beam in which the positron appears in a bound rather than a continuum state with the antiproton. They expect to produce $200 \overline{\mathrm{H}}$ atoms in a first test of this method in 1994 using the E760 hydrogen jet target. The cross-section varies with the $Z^{2}$ of the target gas, and at roughly $1 \mathrm{pb}$ for hydrogen is some $10^{10}$ times below the $\bar{p}-p$ annihilation cross section. The enormous charged particle background will be swept away by bending magnets, leaving the $\overline{\mathrm{H}}$ atoms in a neutral beamline. These 
will subsequently signal their presence by dissociation in a stripper foil, the resulting antiproton and the $\mathrm{e}^{+}$both being detected to give an unambiguous $\overline{\mathrm{H}}$ signal.

A Lamb shift measurement at the $10^{-2}$ precision has been proposed to follow the pilot experiment and would require a sample about $3 \times 10^{4}$ fast $\overline{\mathrm{H}}$ atoms emerging in the neutral beam line. The limitation in the precision obtainable in this measurement is set by the finite transit time (about $10^{-7} \mathrm{~s}$ ) of the $\mathrm{GeV}$ antihydrogen atoms through the Lamb shift apparatus.

\section{Antihydrogen production using metastable antiprotonic helium states}

Another recent proposal is to use the recently discovered metastable antiprotonic helium atoms $[169,170]$ to produce antihydrogen in chemical reactions involving $\mathrm{e}^{+}$or Ps [171]. It has been found that in $3-4$ percent of all exotic atoms formed when $\bar{p}$ stop in helium, the antiproton is able to survive against strong interactions (annihilation) for a time of the order of several $\mu$ sec. This metastability contradicts sharply the longheld belief that all stopped $\bar{p}$ annihilate in less than a picosecond, and is thought to be a property of neutral atoms consisting of an $\alpha$ particle, a $\bar{p}$ in a circular or nearly circular orbital of large angular momentum, and an electron in a $1 S$ state. An antiproton immobilised in such an atom via the formation reaction

$$
\overline{\mathrm{p}}+\mathrm{He} \rightarrow \alpha \overline{\mathrm{p}} \mathrm{e}^{-}+\mathrm{e}^{-} .
$$

may then undergo chemical reactions with $\mathrm{e}^{+}$and Ps to form antihydrogen.

Detailed calculations are being made [172] on the atomic and chemical properties of these metastable states and of the rates of the $\overline{\mathrm{H}}$ forming reactions to clarify questions concerning the screening of both the antiprotonic helium and the Ps by the atomic electrons against penetrating collisions. Other collision-induced processes such as Ps triplet to singlet spin flip ( which destroys the Ps by the faster singlet annihilation rate) and deexcitation of the metastable antiprotonic atom (causing losses of $\bar{p}$ by nuclear capture) must also be considered. The lifetime of $\bar{H}$ against annihilation by collisions with neighbouring helium atoms would be extremely short (perhaps $10^{-10} \mathrm{~s}$ ) in the dense gaseous helium used so far in these experiments, but it may be possible to form the metastable states in the extremely dilute gas which, e.g., constitutes the decelerating medium in the anticyclotron as mentioned before.

\section{Antihydrogen production in a combined $R F+$ Penning trap}

A combination of a Penning trap for the antiprotons with an RF trap (Paul trap) superimposed to confine the positrons was proposed several years ago [173] and further development of this idea was presented at the Munich Workshop [174]. This combined trap has a wider region of stability than the simple Paul trap for two different kinds of particles with a large mass ratio but allows the confinement of both particles in the same volume, thereby increasing the interaction time as compared to that in the nested trap approach [60].

The main disadvantage of the combined trap method is the heating effect of the RF field on both particles. The heating is significant for the positrons and may be balanced by intrinsic radiative cooling; it is less important for the antiprotons and might be counteracted by the cooling effect of the positrons themselves. Thus the recombination rate is 
mainly limited by the micromotion of the positrons.

Assuming $10^{8} \overline{\mathrm{p}} / \mathrm{cm}^{3}$ and $10^{7} \mathrm{e}^{+} / \mathrm{cm}^{3}$ stored at an RF voltage of $\sim 1000 \mathrm{~V}$ and with an average kinetic energy of the positrons of the order of $10 \mathrm{eV}$, this method is estimated [174] to deliver up to $\approx 100 \overline{\mathrm{H}} / \mathrm{s}$ or $10^{5} \overline{\mathrm{H}} /$ day in LEAR conditions. This scheme is now being extended to confine the antihydrogen atoms produced [175] in order to measure their gravitational acceleration.

\section{Antihydrogen production: conclusion}

Among the antihydrogen production schemes discussed above, those based on collisions of antiprotons with positronium or two positrons in a Penning trap are the most promising and comprehensively studied. Those are the techniques which should provide slow, confined antihydrogen for the high-precision spectroscopic studies considered in the present paper. The methods of combined traps and metastable helium states need further studies, especially to demonstrate the feasibility and to solve the problems of antihydrogen confinement.

The main problem with the methods producing antihydrogen beams is that fast $\overline{\mathrm{H}}$ atoms are only available for study during the time they traverse the experimental apparatus, i.e. for less than a few ns. Although this is equivalent to some $10^{6}$ orbital periods (so that the existence of the $\overline{\mathrm{H}}$ atomic state is well defined) it corresponds to only a few transition lifetimes. We cannot, therefore, expect high precision measurements of any physical quantity dependent on these lifetimes unless the number of available atoms in the beam is very large (as is the case for hydrogen beam experiments). A similar argument is valid for the antihydrogen atoms produced from metastable antiprotonic helium owing to the short time of flight between their formation and their first collision with a neighbouring helium atom. A solution to this problem might be to form the metastable states in the much less dense gaseous environment of the anticyclotron, described above in connection with antiproton deceleration methods.

Trapped atoms, on the other hand, are available for many excitation-deexcitation cycles, and the number of atoms required to perform an experiment with a give precision is reciprocally smaller. Experiments have nevertheless been proposed for fast $\overline{\mathrm{H}}$ atoms which use the fact that excitation by passing a beam through a thin foil is much less subject to competition from ionisation than is the case for excitation of trapped atoms [159]. In a Novosibirsk experiment [176] relativistic hydrogen atoms, produced in radiative recombination of $65 \mathrm{MeV}$ protons, were excited to the $2 S$ state by passing them through a thin foil. The Lamb shift was then measured with a precision of $1 \%$ using the Stark effect i.e. periodic $2 S \leftrightarrow 2 P$ transitions in an electric field. There is also a proposal to measure the $1 S$ hyperfine splitting in fast antihydrogen atoms using a combined laser - microwave setup with a precision of $\pm 22.4 \mathrm{ppm}[159]$.

\section{Antihydrogen trapping and cooling}

Trapped $\overline{\mathrm{H}}$ atoms are required for the highest precision antihydrogen spectroscopy experiments discussed above. Moreover, they must be cooled below $1 \mathrm{~K}$ in order to be trapped, and to reduce the Doppler effect from thermal motion. $\overline{\mathrm{H}}$ atoms cannot, of course, be confined in the charged particle trap in which they were produced. However, great advances have recently taken place in the trapping of ordinary atomic hydrogen near 
minima of magnetically inhomogeneous fields [177]. In such cases the required restoring force comes about because the potential energy of the positron's magnetic dipole moment $\vec{\mu}$ in field $\vec{B}$ is $-\vec{\mu} \cdot \vec{B}$. The nucleon moment is small enough to be neglected so this is essentially the potential energy of the entire atom. If $\vec{\mu}$ is antiparallel to $\vec{B}$, the atom will be drawn to magnetic field mimima, where its potential energy is least, while if $\vec{\mu}$ is parallel to $\vec{B}$ the atom will move away from the field minimum. The so-called low-field seekers will therefore be trapped in the potential well near field minimum by such an arrangement. The depth of a trap working on this principle is limited by achievable magnetic field gradients. Fields of strength about $1 \mathrm{~T}$ can in fact be produced with gradients large enough to trap low-field seeking hydrogen atoms at energies up to $0.67 \mathrm{~K}$ (corresponding to about $55 \mu \mathrm{eV}$ ), and this sets a corresponding limit on the temperature to which the antiprotons and positrons must be cooled before recombination can be attempted. The simplest (anti)hydrogen trap is a magnetostatic, two-coil quadrupole which can be enclosed inside a charged particle trap.

The simple quadrupole trap described above is not normally used for hydrogen trapping as there is a $\vec{B}=0$ region which permits Majorana transitions to untrapped (i.e. high-field seeking) states. Ioffe-type traps, i.e. pure quadrupole or combined quadrupoleoctupole magnetic traps (see, e.g., Fig. 17) seem to be satisfactory for $T<1 \mathrm{~K}$ antihydrogen for all experiments, because they contain no $\vec{B}=0$ region and have a shallow potential valley which does not induce too many $\overline{\mathrm{H}}-\overline{\mathrm{H}}$ collisions at these very low temperatures $[177$, 178]. Large numbers of hydrogen atoms have successfully been confined in Ioffe-type traps at Amsterdam and MIT.

Even at energies equivalent to $1 \mathrm{~K}$ temperature, antihydrogen atoms would still be too energetic for extremely high precision spectroscopic measurements, for which $\mathrm{mK}$ temperatures are required. In the hydrogen case, this can be achieved by evaporation of the higher energy atoms [179], and this way atomic hydrogen was cooled to $100 \mu \mathrm{K}$ at a density of $8 \times 10^{13}$ atoms $/ \mathrm{cm}^{3}$ at MIT [180]. This requires atomic number densities of order $10^{10} / \mathrm{cm}^{3}$, and is clearly out of the question with antihydrogen, given the small number of these atoms which are likely to be available. Cooling by mixing with cold antiprotons has also been considered, but this would be very slow: the time to cool from $T=1 \mathrm{~K}$ to $T=10 \mathrm{mK}$ is estimated to be of the order of an hour [177] for realistic numbers of trapped particles.

Laser cooling must, therefore, be developed for cooling the trapped antihydrogen atoms [179]. Applied to free (i.e. untrapped) atoms this method would require three orthogonal Lyman $-\alpha$ laser beams, detuned slightly below the $1 S-2 P$ resonance. An $\overline{\mathrm{H}}$ atom moving against one of the beams (on resonance) absorbs a photon from the beam and re-radiates it isotropically. When the $\overline{\mathrm{H}}$ is moving in the opposite direction, no absorption takes place because its motion puts the $\overline{\mathrm{H}}$ atom further off resonance. This arrangement is known as optical molasses and does not itself constitute a trap, but only a region in which the atom diffuses slowly while cooling. For the case being considered where the atoms are already confined in a magnetic trap, only one beam would be required [177]. Optical cooling can be combined with adiabatic cooling, i.e. by slowly reducing the trap depth. In this case, two more orders of magnitude can be gained in the final temperature if starting with a deep enough trap [181]. All these techniques, including beam deceleration with a laser continuously tuned to follow the beam velocity are being or have been tested with 
Figure 17: Scenario for antihydrogen spectroscopy in a Ioffe-type trap [7]. Two UV laser pulses lift the $\overline{\mathrm{H}}$ atoms to the $2 S$ level, the $2 P 1 / 2$ levels are quenched with microwaves via the spontaneously decaying $2 P 3 / 2$ state. The Lyman- $\alpha$ photons can be detected from the spontaneous $2 S-1 S$ transition.

atomic beams. The main problem, however, is to provide the high-powered, hydrogen Lyman $-\alpha$ lasers for antihydrogen manipulation, although there are proposals for building them $[177,179,181]$.

\section{SPECTROSCOPY OF ANTIHYDROGEN}

As the spectroscopic properties of antihydrogen are expected to be similar if not identical to those of hydrogen, most of the principles of high precision hydrogen spectroscopy are also valid for antihydrogen. A fully comprehensive treatment of such techniques is beyond the scope of this article, and would in any case be out of place. We therefore confine our remarks in what follows to consideration of the few additional constraints on antihydrogen spectroscopy which are not present in the hydrogen case, and to a discussion of several transitions which might be candidates for initial experiments on antihydrogen. As we have seen above, the $1 S-2 S$ transition is paramount among these.

One important practical difference which faces the antihydrogen spectroscopists is that the number of atoms likely to be available for spectroscopy is several orders of magnitude smaller than the smallest number normally used in hydrogen experiments. This challenges them to develop methods which combine extreme detection sensitivity with the highest possible resolution. We assume that a cold sample of at least $10^{3}$ antihydrogen atoms has been produced by recombination of antiprotons and positrons (or positronium) at $4 \mathrm{~K}$. However, as discussed above, several techniques have been proposed to produce unconfined antihydrogen atoms and we shall briefly consider experiments which can be done with those. Two possibilities are then conceivable:

- the antihydrogen atoms are stored in a magnetic trap and are available for spectroscopy over a time period of several minutes or longer;

- the atoms are not trapped and the optical excitation has to take place in the first $3 \mu \mathrm{s}$ after the recombination (the mean velocity at $4 \mathrm{~K}$ is $300 \mathrm{~m} / \mathrm{s}$ ); this could be the case for antihydrogen produced from metastable helium states. 
Since all of the known techniques of trapping positrons, antiprotons or neutral atoms make use of strong magnetic fields, Zeeman and Doppler broadening are expected to be dominant systematic effects. At very high resolution, with a projected line width of the order of several ten $\mathrm{kHz}$, the transit time broadening and the relativistic Doppler effect may contribute as well.

The atomic energy levels may be observed by exciting a one-photon or a twophoton electric dipole transition (the single-photon excitation rate is larger by orders of magnitude). Single-photon spectroscopy is possible, however, between energy levels with different Zeeman effects only. Thus, in addition to the Doppler effect one should expect a strong Zeeman broadening (up to several $\mathrm{GHz}$ ) in inhomogeneous magnetic fields. On the contrary, the two-photon excitation is intrinsically Doppler-free and may be applied between levels of equal Zeeman shifts so that the effect of magnetic field inhomogeneities could be neglected. However, the poor excitation rate restricts its application and it appears to be feasible only for trapped antihydrogen or at very high laser intensities $\left(>5000 \mathrm{Wcm}^{-2}\right.$ ), see Fig. 17. The excited atoms could be detected either by observing the fluorescence of the radiative decay to the ground state by a photomultiplier or via photo ionization with an additional laser beam. Detection efficiencies are of the order of $10^{-2}$ to $10^{-4}$. In the following we consider the various possible transitions in detail.

\section{Spectroscopy from the ground state}

The energy interval of $2468 \mathrm{THz}$ between the $n=1$ and the $n=2$ states of antihydrogen is determined mainly by the antiRydberg constant (i.e. the mass and charge of the positron). The reduced mass correction contributes at the $0.5 \%$ level and contains information about the mass ratio of the positron and the antiproton. At the ppm-level the $1 S$ Lamb shift comes into play, including radiative corrections and the vacuum polarization. The influence of the finite nuclear charge radius appears at the $10^{-9}$ level.

\section{One-photon $1 S \rightarrow 2 P$ transition (Lyman- $\alpha$ )}

The dominant line broadening at $4 \mathrm{~K}$ is caused by the Doppler effect which is of the order of $2 \mathrm{GHz}$. If colder atoms are confined in a magnetic trap one has to accept a large Zeeman-broadening of about $5 \mathrm{GHz}$ at $100 \mathrm{mK}$. Even if the atoms could be cooled down to the Doppler limit $(2 \mathrm{mK})$ the resolution would in principle be limited to $4 \times 10^{-8}$ because of the large natural linewidth of about $100 \mathrm{MHz}$. No continuous wave laser is available at the excitation wavelength of $121 \mathrm{~nm}$, but pulsed coherent radiation may be generated by four-wave mixing in a noble gas. In Amsterdam Walraven and coworkers $[182,183]$ were able to observe this transition in hydrogen in a magnetic trap. They also demonstrated Doppler-cooling of trapped hydrogen, down to several $\mathrm{mK}$. Using Lyman $-\alpha$ radiation, experimental difficulties may arise from the complexity of the laser source and the relatively energetic ultraviolet radiation which can possibly damage the optical components. The excitation rate is high enough to allow for the spectroscopy of untrapped atoms. However, the production of antihydrogen has to be matched to the laser pulse length of a few ns and to its repetition rate of about $100 \mathrm{~Hz}$. 


\section{Two-photon $1 S \rightarrow 2 S$ transition}

The strongest single-photon transition between the $2 S$ and $1 S$ levels is a magnetic dipole one, which is allowed in the Dirac approximation. However, its rate is about $\alpha^{6}$ times the typical allowed electric dipole rate [151, 152],

$$
\Gamma=\alpha^{11} \frac{m c^{2}}{2^{4} 3^{3} \hbar}=5.6 \times 10^{-6} \mathrm{~s}^{-1}
$$

i.e. about 1 event in 2 days. The emission of two electric dipole photons is faster, $\sim \alpha^{3}$ times typical allowed dipole rates, i.e. about $7 \mathrm{~s}^{-1}[151,153,154]$.

The extremely small natural linewidth $(1 \mathrm{~Hz})$ corresponding to the long lifetime of the metastable $2 S$ state makes this unique transition the most promising candidate for high precision measurements. Doppler-free excitation is possible by the absorption of two photons from opposite directions (two-photon spectroscopy of trapped hydrogen atoms is discussed in detail by Kleppner in [155]). In sharp contrast to the one photon excitation, the relativistic Zeeman-shift due to the magnetic field is only $18 \mathrm{~Hz} /$ Gauss (at least for transitions between the $m_{F}= \pm 1$ states). However, the poor excitation rate $\left(\Gamma=3.4 I^{2} / \Delta \nu\right.$ in units $\mathrm{W}^{-2} \mathrm{~cm}^{4} \mathrm{~s}^{-1}$ with light intensity $I$ and line width $\left.\Delta \nu\right)$ permits this kind of spectroscopy with trapped atoms only.

Spectroscopy of the $1 S-2 S$ transition inside a magnetic trap is under preparation at MIT where [180] where hydrogen atoms are trapped at $30 \mu \mathrm{K}$ and at a density of $8 \times 10^{13}$ atoms $/ \mathrm{cm}^{3}$. The ultimate resolution depends mainly on the temperature inside the trap [155]:

$$
\Delta \nu=\frac{\alpha^{2}}{4} \frac{k T}{h}
$$

giving $300 \mathrm{kHz} / \mathrm{K}$ i.e. at a temperature of $3 \mathrm{mK}$ (as obtained by optical cooling in the Amsterdam experiment $[182,183]$ ) the expected linewidth is about $\Delta \nu=900 \mathrm{~Hz}$. Better resolution will be possible in stiff traps where a steep magnetic field gradient confines the atoms to a small volume. Motional averaging then leads to a reduction of the line width [155] by a factor of 25 for a field gradient of $1 \mathrm{~T} / \mathrm{cm}$ and a temperature of $3 \mathrm{mK}$.

The excitation wavelength of $243 \mathrm{~nm}$ may be generated by frequency doubling of a dye laser in a nonlinear crystal. In a dense atomic hydrogen beam $\left(10^{9}\right.$ atoms $\left./ \mathrm{cm}^{3}\right)$ this transition has been observed with a resolution of $12 \mathrm{kHz}$ [184].

The frequency of the $1 S-2 S$ transition in hydrogen has been measured recently with a precision of $1.8 \times 10^{-11}$ by comparing the frequency of the excitation light at $1234 \mathrm{THz}$ with that of a methane stabilized helium-neon laser at $88.5 \mathrm{THz}$ [6]. The laser frequency was calibrated relative to the cesium standard with an accuracy of parts in $10^{12}$.

\section{Spectroscopy from excited states}

As discussed above, double collisions of $\bar{p}$ and $\mathrm{e}^{+}$will produce antihydrogen atoms in highly excited Rydberg states. Almost all of these will decay in a series of radiative transitions into the ground state. However a small fraction will end up in the metastable $2 S$ state [165]. These may be used for excited state spectroscopy. The energy intervals to the next highest excited states are small, permitting the use of easily available laser sources. Continuous wave excitation with high transition probability should be possible. 
The natural linewidths are about an order of magnitude smaller than that of the Lyman- $\alpha$ transition.

\section{One-photon transition from $2 S$ to $3 P$ and $4 P$}

These transitions offer a good compromise between excitation probability and resolution. The natural linewidths of several tens of MHz may be resolved by a commercially available, continuous-wave laser equipment (ion-laser pumped dye laser). However, the large Doppler broadening (600 $\mathrm{MHz}$ at $4 \mathrm{~K}$ ) still causes problems. Doppler-free techniques, such as saturation spectroscopy or polarisation spectroscopy, seem difficult. In a trap there exists no closed optical cycle, so that most of the atoms decay into the ground state and are no longer available for spectroscopy. The excitation rates should be high enough to observe untrapped atoms. For these frequencies (as opposed to the Lyman$\alpha$ transitions) continuous laser sources are available which could make good use of the continuous schemes of antihydrogen production. In hydrogen these transitions have been studied in an atomic beam configuration by W. Lichten and coworkers [185]. For an absolute frequency measurement, the $2 \mathrm{~S}-4 \mathrm{P}$ transition is advantageous, as a well-calibrated, portable optical secondary standard exists at the transition wavelength near $486 \mathrm{~nm}$ which is based on a narrow $(10 \mathrm{MHz})$ resonance line of the ${ }^{130}$ Te molecule and has an accuracy better than $1 \mathrm{MHz}$ [186]. A relative precision of $10^{-6}$ to $10^{-8}$ is attainable.

\section{Two-photon transition from $2 S$ to $4 S$}

With two photon excitation this resonance transition is Doppler-free, but the small excitation rate excludes an excitation of untrapped atoms, while for trapped atoms, as we have noted, no closed optical cycle exists. Although this transition has been successfully used in hydrogen spectroscopy [184] it does not seem to be a reasonable tool to investigate antihydrogen, at least for the first generation of experiments.

\section{Transitions between Rydberg states}

For completeness we should mention the possibility of radio frequency spectroscopy between Rydberg states. During the decay cascade immediately after the recombination, the highly excited antihydrogen atom undergoes a series of transitions between circular Rydberg states. These states have millisecond lifetimes and allow in principle the Rydberg constant to be measured with a precision of $10^{-9}$. Two hydrogen-beam experiments are planned along these lines [187, 188]. There are no results yet and it is unclear whether this concept is applicable for the restrictive conditions under which antihydrogen may be produced.

\section{Spectroscopy during recombination}

As mentioned in Chapter 3 , it is possible to stimulate the radiative recombination of antihydrogen into a Rydberg state of $n=12$ or $n=11$. The recombination rate may be enhanced by several orders of magnitude but is limited by an unavoidable ionisation by the same stimulating light field. This ionisation rate may be reduced by an additional laser which stimulates a transition from the Rydberg state to a lower lying energy level with $n=$ 3 or $n=2$. As a result, the Rydberg state is rapidly depopulated before the antihydrogen 
atom is lost via ionisation. The related increase of antihydrogen production rate may be used to detect the depopulating transition and offers a novel way of inverse spectroscopy. The transition from $n=11$ to $n=3$ near $886 \mathrm{~nm}$ may be stimulated even with a semiconductor laser whereas for the transition to the $n=2$ state a titanium sapphire laser may be used to drive a Doppler-free and Zeeman-free, two-photon transition near $752 \mathrm{~nm}$. Since antihydrogen atoms may be detected with high efficiency by observing the antiproton annihilation radiation, and since it does not require trapped antihydrogen atoms, the proposed inverse two-photon spectroscopy might possibly be the most promising tool for the first generation of high-precision measurements.

\section{CONCLUSION}

Over the last eight years an impressive number of new developments have come together that have changed the prospects of antihydrogen production from a pipe dream to a realistic possibility. In 1985 ideas of antihydrogen and hydrogen atom trapping and cooling were considered to be somewhat wild, and the $1 S-2 S$ spectroscopy of hydrogen had only been accomplished with MHz resolution. Now, in 1994, more than $10^{5}$ antiprotons are regularly trapped at cryogenic temperatures and stored for periods of months; up to $10^{14}$ hydrogen atoms can be trapped for hours at a time; laser cooling of atoms is extensively used to reach $\mu \mathrm{K}$ temperatures; and $1 S-2 S$ resolution has dropped to $\mathrm{kHz}$, with improvement expected soon to a few hundred $\mathrm{Hz}$. It is therefore now possible to propose seriously the combination of these techniques with the object of producing cold, trapped antihydrogen atoms for spectroscopic studies at LEAR. Much further work remains to be done on the various recombination reactions, and on the development of lasers that will be required for antihydrogen cooling. Nevertheless, optical spectroscopy of the $1 S-2 P$ and $2 S-3 P / 4 P$ transitions would be possible, even on untrapped antihydrogen, and for trapped antihydrogen the high precision spectroscopy allowed by the two-photon $1 S-2 S$ transition can be confidently anticipated. The ability to compare this energy interval with very high precision in hydrogen and antihydrogen is a powerful new tool that will permit unique tests of CPT symmetry and the weak equivalence principle for antiparticles. In the case of CPT, it will be possible to test this symmetry for the first time for a fundamental atomic constant (the Rydberg), with an ultimate precision close to that of the comparison of the neutral kaon masses. It will also be possible to derive the first CPT tests of the antiproton's mass and charge separately, with the level of precision reached in the electron-positron $g$-factor comparisons. The first direct test of WEP for an antiparticle (the positron) will be possible, and should achieve a precision equivalent to similar tests on the constituents of ordinary matter. Antihydrogen formation at LEAR will extend the tests of fundamental principles that have been accomplished with hydrogen, positronium and muonium into the antimatter world.

\section{ACKNOWLEDGEMENTS}

This review has resulted from a number of antihydrogen working group meetings held at CERN in the last two years and an Antihydrogen Workshop in Munich, 3031 July 1992. These events were organised in response to a request in 1990 from the Super Proton Synchrotron and LEAR Committee (SPSLC) of CERN for an evaluation of the physics potential of the antihydrogen atom. We would like to thank all of our 
colleagues (too numerous to list here) who participated in those meetings and thereby provided material for the present review. We also thank Profs. Michael H. Holzscheiter, Pierre Lefèvre, Akihiro Mohri and Gabriele Torelli for illuminating discussions and for communicating unpublished recent results and Prof. Daniel Kleppner whose careful and constructive criticism of the original manuscript resulted in many improvements.

\section{References}

[1] P. A. M. Dirac, Proc. Roy. Soc. A117 (1928) 610.

[2] P. A. M. Dirac, Proc. Roy. Soc. A133 (1931) 60.

[3] B. I. Deutch: Hyp. Int. 73 (1992) 175.

[4] R.J. Hughes, Nature 353 (1991) 700.

[5] D. H. McIntyre and T. W. Hänsch, Metrologia 25 (1988) 61.

[6] T. Andraea, W. König, R. Wynands, D. Leibfried, F. Schmidt-Kaler, C. Zimmermann, D. Meschede, T. W. Hänsch, Phys. Rev. Lett. 69 (1992) 1923.

[7] T. W. Hänsch, C. Zimmermann, In: Antihydrogen, Proc. Antihydrogen Workshop, Munich, 1992 (J. Eades, ed.); Hyp. Int. 76 (1993) 47.

[8] C. D. Anderson, Science 76 (1932) 238; Phys. Rev. 43 (1933) 491.

[9] G. Lüders, Det. Kon. Dan. Vid. Sel. Mat.-fys. Medd. 28, no. 5, pg. 1 (1954); G. Lüders, Ann. Phys. (NY) 2 (1957) 1.

[10] W. Pauli in "Niels Bohr and the development of physics," W. Pauli, ed. (Pergamon, New York, 1955), p. 30.

[11] J. S. Bell, Proc. Roy. Soc. A231 (1955) 479.

[12] R. Jost, "The general theory of quantised fields," (American Mathematical Society, Providence Rhode Island, 1965).

[13] R. Carosi et al., Phys. Lett. B237 (1990) 303.

[14] R. S. VanDyck, Jr., P. B. Schwinberg, and H. G. Dehmelt, Phys. Rev. Lett. 59 (1987) 26.

[15] G. Gabrielse, X. Fei, L. A. Orozco, R. L. Tjoelker, J. Haas, H. Kalinowsky, T. A. Trainor, W. Kells, Phys. Rev. Lett. 65 (1990) 1317.

[16] M. Kobayashi, A. I. Sanda, "On testing the CPT symmetry", RU 92/3/8, Rockefeller Univ., 1992.

[17] B. W. Petley, "The fundamental physical constants and the frontier of measurement", (Adam Hilger, Bristol, 1988).

[18] A. I. Oksak and I. T. Todorov, Comm. Math. Phys. 11 (1968) 125;

D. T. Stoyonov and I. T. Todorov, J. Math. Phys. 9 (1968) 2146.

[19] R. M. Wald, Phys. Rev. D 21 (1980) 2742.

[20] P.K. Kabir, Phys. Rev. D 2 (1970) 540.

[21] R. J. Hughes and B. I. Deutch, Phys. Rev. Lett. 69 (1992) 578.

[22] Particle Data Group, Phys. Rev. D11 (1992) S1.

[23] M. Marinelli and G. Morpurgo, Phys. Lett. B137 (1984) 439.

[24] P. B. Schwinberg, R. S. VanDyck and H. G. Dehmelt, Phys. Lett. 81A (1981) 119.

[25] F. Hoyle, Proc. Roy. Soc. A257 (1960) 431.

[26] S. Chu, A. P. Mills and J. L. Hall, Phys. Rev. Lett. 52 (1984) 1689;

K. Danzmann, M. S. Fee and S. Chu, Phys. Rev. A39 (1989) 6072. 
[27] D. J. Heinzen and D. J. Wineland, Phys. Rev. A42 (1990) 2977.

[28] S. Chu, in The Hydrogen Atom, G. F. Bassani et al., eds., (Springer, New York, 1989), pg. 144.

[29] P. F. Winkler et al., Phys. Rev. A5 (1972) 83.

[30] A. Kreissl et al., Z. Phys. C37 (1988) 557.

[31] W. Quint, G. Gabrielse, In: Antihydrogen, Proc. Antihydrogen Workshop, Munich, 1992 (J. Eades, ed.); Hyp. Int. 76 (1993) 379.

[32] S. Chu et al., Phys. Rev. Lett. 60 (1988) 101.

[33] A. Einstein, Jahrb. Rad. Elektr 4 (1907) 411.

[34] A. Einstein (1920) quoted in, A. Pais: "Subtle is the lord" (OUP, Oxford, 1982), pg. 178.

[35] C. M. Will, "Theory and Experiment in Gravitational Physics", (Cambridge University Press, Cambridge, 1981).

[36] L. I. Schiff, Am. J. Phys. 28 (1960) 340.

[37] R. v. Eötvös, D. Pekar and E. Fekete, Ann. Phys. (Leipzig) 68 (1922) 11.

[38] E. G. Adelberger, Phys. Rev. D42 (1990) 3267.

[39] T. E. O. Ericson and A. Richter, Europhys. Lett. 11 (1990) 295.

[40] R. J. Hughes: "The Equivalence Principle", Report LA-UR-93-3827, Los Alamos, 1993 (to be published in Contemporary Physics).

[41] J. C. Maxwell, Phil. Trans. Roy. Soc. 155 (1865) 459, section 82.

[42] H. A. Lorentz, Proc. Amst. Acad. 2 (1900) 559.

[43] O. Heaviside, "Electromagnetic theory," 3rd edn., vol. 1, p. 455 (Chelsea, New York, 1971).

[44] A. Schuster, Nature 58 (1898) 367, 618.

[45] T. Goldman, R.J.Hughes and M.M. Nieto, Phys. Lett. B171 (1986) 217.

[46] E. G. Adelberger et al., Phys. Rev. Lett. 66 (1991) 850.

[47] P. Morrison, Am. J. Phys. 26 (1958) 358.

[48] A. Einstein, Ann. Phys. (Leipzig) 35 (1911) 898.

[49] K. Nortvedt, Phys. Rev. D11 (1975) 245.

[50] R. J. Hughes, Am. J. Phys. 58 (1990) 826.

[51] J. S. Bell in: "Fundamental symmetries", P. Bloch et al., eds., (Plenum, New York, 1987), pg. 1.

[52] G. Nordström, Ann. Phys. (Leipzig) 40 (1913) 856;

A. L. Harvey, Am. J. Phys. 33 (1965) 449; R. H. Dicke, Ann. Phys. (NY) 31 (1965) 235.

[53] R. V. Pound and G. A. Rebka, Phys. Rev. Lett. 4 (1960) 337.

[54] W. G. Unruh and G. I. Opat, Am. J. Phys. 47 (1979) 743.

[55] R. J. Hughes, Phys. Rev. D41 (1990) 2367.

[56] P. Thieberger, Nuovo Cim. 35 (1965) 688.

[57] E. F. Beall, Phys. Rev. 1 (1970) 961.

[58] R. J. Hughes, in: "New and Exotic Phenomena '90", O. Fackler and J. Tranh Thanh Van eds., (Editions Frontières, Gif-sur-Yvette, 1990), pg. 263.

[59] R. J. Hughes and M. H. Holzscheiter, J. Mod. Opt., 39 (1992) 263.

[60] G. Gabrielse, S. R. Rolston, L. Haarsma, W. Kells, Phys. Lett. A 129 (1988) 38. 
[61] N. Beverini, V. Lagomarsino, G. Manuzio, F. Scuri, G. Torelli, Hyp. Int. 44 (1988) 357.

[62] L. Koester, Phys. Rev. D14 (1976) 907;

I. Esterman et al., Phys. Rev. 71 (1947) 238.

[63] F. C. Witteborn, W. M. Fairbank, Phys. Rev. Lett. 19 (1967) 1049.

[64] W. E. Thirring, Ann. Phys. (NY) 16, 96 (1961).

[65] J. P. Turneaure et al., Phys. Rev. D27, 1705 (1983).

[66] M. H. Holzscheiter, In: "LEAP'90", Proc. First Biennial Conf. on Low Energy Antiproton Physics, Stockholm, 1990, P. Carlson et al., eds., World Scientific, 1991, p. 437.

[67] L. I. Schiff, Proc. Nat. Acad. Sci. 45 (1959) 69.

[68] R. J. Hughes, In: Antihydrogen, Proc. Antihydrogen Workshop, Munich, 1992 (J. Eades, ed.); Hyp. Int. 76 (1993) 3.

[69] M. L. Good, Phys. Rev. 121 (1961) 311.

[70] J. S. Bell and J. K. Perring, Phys. Rev. Lett. 13 (1964) 348.

[71] I. R. Kenyon, Phys. Lett. B237 (1990) 274.

[72] R. J. Hughes, Phys. Rev. D 46 (1992) R2283.

[73] W. E. Thirring, in Particles, Currents and Symmetries, P. Urban ed., (Springer, New-York, 1968), Acta. Phys. Aust. Suppl. V (1968) 391;

Essays in Physics 4 (1972) 125.

[74] M. von Laue, Ann. Phys. (Leipzig) 35 (1911) 524.

[75] R. J. Hughes and M. H. Holzscheiter, Phys. Rev. Lett. 66 (1991) 854.

[76] E. Fischbach and C. Talmadge, Nature 356 (1992) 207.

[77] E. P. Wigner, Proc. Am. Phil. Soc. 93 (1949) 521; S. Nussinov, Phys. Rev. Lett. 59 (1987) 2401.

[78] P. Rastall, Can J. Phys. 38 (1960) 975.

[79] M. Milgrom, Ap. J. 270 (1983) 365.

[80] R. H. Sanders, Astron. Astrophys. 136 (1984) L21; 154 (1986) 135.

[81] D. G. Boulware and S. Deser, Phys. Rev. D6 (1972) 3368.

[82] P. Lefevre, In: "Physics at LEAR with low energy antiprotons", Proc. 4th LEAR Workshop, Villars-sur-Ollon, 1987, p. 19;

M. Chanel, In: "LEAP'90", Proc. First Biennial Conf. on Low Energy Antiproton Physics, Stockholm, 1990, P. Carlson et al., eds., World Scientific, p. 470 (1991);

S. Baird, J. Bosser, M. Chanel, P. Lefevre, R. Rey, D. Manglunki, S. Maury, D. Möhl, G. Tranquille, In: Antihydrogen, Proc. Antihydrogen Workshop, Munich, 1992 (J. Eades, ed.); Hyp. Int. 76 (1993) 61.

[83] N. Beverini, V. Lagomarsino, G. Manuzio, F. Scuri, G. Torelli, Hyp. Int. 44 (1988) 209.

[84] H. Kalinowsky, In: Antihydrogen, Proc. Antihydrogen Workshop, Munich, 1992 (J. Eades, ed.); Hyp. Int. 76 (1993) 73.

[85] J. Eades et al.: "A general purpose ultra low energy antiproton facility for LEAR”, CERN/PSCC/89-15, PSCC/P 118, 10 May 1989;

J. Eades, L. M. Simons: Nucl. Instr. Meth. A278 (1989) 368. 
[86] A. Coc et al., In: "LEAP'90", Proc. First Biennial Conf. on Low Energy Antiproton Physics, Stockholm, 1990, P. Carlson et al., eds., World Scientific, 1991, p. 431.

[87] G. Gabrielse, W. Jhe, D. Phillips, W. Quint, C. Tseng, L. Haarsma, K. Abdullah, J. Gröbner, H. Kalinowsky, In: Antihydrogen, Proc. Antihydrogen Workshop, Munich, 1992 (J. Eades, ed.); Hyp. Int. 76 (1993) 81.

[88] G. Gabrielse, X. Fei, L. A. Orozco, R. L. Tjoelker, J. Haas, H. Kalinowsky, T. A. Trainor, W. Kells, Phys. Rev. Lett. 63 (1989) 1360.

[89] L. M. Simons, Physica Scripta T22, 90 (1988).

[90] E. C. Aschenauer et al., "Report to the SPSL Committee on the beam tests made by the P118 collaboration at LEAR", CERN/SPSLC/92/28, SPSLC/M494, 20 May 1992.

[91] P. DeCecco, P. Hauser, D. Horváth, F. Kottmann, J. Missimer, L. M. Simons, D. Taqqu, D. Abbot, B. Bach, R. T. Siegel, D. Viel, In: Antihydrogen, Proc. Antihydrogen Workshop, Munich, 1992 (J. Eades, ed.); Hyp. Int. 76 (1993) 275.

[92] L. S. Brown, G. Gabrielse, Rev. Mod. Phys. 58 (1986) 233.

[93] A. Wolf, H. Poth, W. Schwab, B. Seligmann, M. Wörtge, H. Haseroth, C. E. Hill, J.-L. Vallet, Hyp. Int. 44 (1988) 217;

S. L. Rolston, G. Gabrielse, Hyp. Int. 44 (1988) 233;

N. Beverini, V. Lagomarsino, G. Manuzio, F. Scuri, G. Testera, G. Torelli, Hyp. Int. 44 (1988) 247.

[94] M. H. Holzscheiter et al., "Tests on a large Penning trap at LEAR", LEAP'92, Second Biennial Conf. on Low-Energy Antiproton Physics, Courmayeur, Aosta Valley, Italy, 1992; Nucl. Phys. A558 (1993) 679c;

M. H. Holzscheiter et al., "Progress report on PS200", CERN SPSLC 92-64, SPSLC M-514, 1992.

[95] M. H. Holzscheiter: private communication.

[96] P. J. Schultz, K. G. Lynn, Rev. Mod. Phys. 60 (1988) 701.

[97] M. Charlton, G. Laricchia, In: Antihydrogen, Proc. Antihydrogen Workshop, Munich, 1992 (J. Eades, ed.); Hyp. Int. 76 (1993) 97.

[98] A. P. Mills, L. Pfeiffer, Phys. Rev. B32 (1985) 53.

[99] J. Dahm, R. Ley, K. D. Niebling, R. Schwarz, G. Werth, Hyp. Int. 44 (1988) 151.

[100] P. J. Schultz, K. G. Lynn, Phys. Rev. B 26 (1982) 2390.

[101] A. P. Mills, Jr., E. D. Shaw, R. J. Chichester, D. M. Zuckerman, Phys. Rev. B 40 (1989) 2045.

[102] S. Chu, A. P. Mills, Phys. Rev. Lett. 48 (1982) 1333.

[103] G. Gabrielse, presented at Workshop on Positron Interactions with Atoms, Molecules and Clusters, Bielefeld, Germany, July 1993.

[104] D. T. Britton, P. A. Huttunen, J. Makinen, E. Soininen, A. Vehanen, Phys. Rev. Lett. 62 (1989) 2413;

P. A. Huttunen et al., Phys. Rev. B 42 (1990) 1560.

[105] N. Zafar, G. Laricchia, M. Charlton, T. C. Griffith, Hyp. Int. 73 (1992) 213.

[106] R. H. Howell, R. A. Alvarez, M. Stanek, Appl. Phys. Lett. 40 (1982) 751. 
[107] A. Mohri, T. Michishita, T. Yuyama, H. Tanaka, Jap. J. Appl. Phys. 30 (1991) L936

[108] W. R. Nelson, H. Hirayama, D. W. O. Rogers, "The EGS4 code system", SLAC265, Stanford, 1985.

[109] A. Mohri: Private communication.

[110] A. P. Mills, Jr. et al., Phys. Rev. Lett. 67 (1990) 575;

A. P. Mills, Jr.: Appl. Phys. 23 (1980) 189.

[111] F. M. Jacobsen, M. Charlton, J. Chevallier, B. I. Deutch, G. Laricchia, J. Appl. Phys. 67 (1990) 575.

[112] M. R. Poulsen, M. Charlton, J. Chevallier, B. I. Deutch, L. V. Jorgensen, G. Laricchia, J. Phys. Condens. Matt. 3 (1991) 2849.

[113] R. Paulin, G. Ambrosino, J. Phys. (Paris) 29 (1968) 263.

[114] W. Brandt, R. Paulin, Phys. Rev. Lett. 21 (1968) 193.

[115] S. Curry, A. L. Schawlow, Phys. Lett. 37 A (1971) 5.

[116] K. P. Ziock, R.H.Howell, R.Magnotta, R.A. Failor, K.M. Jones, Phys. Rev. Lett. 64 (1990) 2366.

[117] T. E. Cowan et al., Nucl. Instr. Meth. B 56/57 (1991) 599.

[118] C. M. Surko, M. leventhal, A. Passner, Phys. Rev. Lett. 62 (1989) 901.

[119] T. J. Murphy, C. M. Surko, Phys. Rev. A46 (1992) 5696.

[120] R. S. Conti et al., Nucl. Instr. Meth. A 299 (1990) 420;

R. S. Conti, B. Ghaffari, T. D. Steiger, In: Antihydrogen, Proc. Antihydrogen Workshop, Munich, 1992 (J. Eades, ed.); Hyp. Int. 76 (1993) 127.

[121] A. P. Mills, Jr., Appl. Phys. 22 (1980) 273

[122] L. Haarsma, K. Abdullah, G. Gabrielse, In: Antihydrogen, Proc. Antihydrogen Workshop, Munich, 1992 (J. Eades, ed.); Hyp. Int. 76 (1993) 143. G. Gabrielse, B. L. Brown, in: "Hydrogen Atom", G. F. Bassani et al., eds. (Springer, Berlin, 1989), p. 196.

[123] D. J. Wineland, C. S. Weimer, J. J. Bollinger, In: Antihydrogen, Proc. Antihydrogen Workshop, Munich, 1992 (J. Eades, ed.); Hyp. Int. 76 (1993) 115.

[124] P. Lefèvre: Private communication.

[125] G. I. Budker, A. N. Skrinskii: Sov. Phys. Uspekhi 21 (1978) 277.

[126] H. Herr, D. Möhl, A. Winnacker: In "Proc. Workshop on Physics at LEAR with Low-Energy Cooled Antiprotons", U. Gastaldi, R. Klapisch, eds., Plenum, New York, 1984, p. 659.

[127] E.C.G. Stueckelberg, P.M. Morse: Phys. Rev. 35, (1930) 16.

[128] W. Wessel: Ann. Phys. (Leipzig) 5 (1930) 611.

[129] D. R. Bates et al.: Proc. Roy. Soc. (London) A710 (1939) 322.

[130] A. Burgess: Mon. Not. R. Astro. Soc. 118 (1958) 477.

[131] M. J. Seaton: Mon. Not. R. Astro. Soc. 118 (1958) 477.

[132] H. A. Bethe, E. E. Salpeter: Quantum Mechanics of One- and Two-Electron Atoms, Plenum, New York, 1977, p. 321.

[133] V. B. Berestetskii, E. M. Lifshitz, L. P. Pitaevskii: Quantum Electrodynamics, 2nd Ed., Pergamon, Oxford, 1982, p. 211.

[134] L. Spitzer: Physics of Fully Ionized Gases, Interscience, New York, 1956.

[135] M. Bell, J. S. Bell: Part. Acc. 12 (1982) 49. 
[136] V. M. Katkov, V. M. Strakhovenko: Sov. Phys. JETP 48 (1979) 639.

[137] E. M. Lifshitz, L. P. Pitaevskii: Physical Kinetics, Pergamon, Oxford, 1981, p. 105.

[138] R. Neumann, H. Poth, A. Winnacker, A. Wolf, Z. Physik A 313 (1983) 253.

[139] G. Gabrielse et al.: In Proc. First Workshop on Antimatter Physics at Low Energy, FNAL, 1986, p. 211.

[140] B. I. Deutch et al:: In Proc. First Workshop on Antimatter Physics at Low Energy, FNAL, 1986, p. 211.

[141] D. R. Bates: Adv. At. Mol. Phys. 20 (1985) 1.

[142] D. R. Bates, A. E. Kingston, R. W. P. McWirther: Proc. Roy. Soc. (London) A267 (1962) 297.

[143] B. Makin, J. C. Keck: Phys. Rev. Lett. 11 (1963) 281.

[144] P. Mansback, J. C. Keck: Phys. Rev. 181 (1969) 275.

[145] D. R. Bates, I. Mendas: J. Phys. B 8 (1975) 1770.

[146] J. Stevefelt et al.: Phys. Rev. A 12 (1975) 1246.

[147] E. S. Chang: Phys. Rev. A 31 (1985) 495.

[148] M. E. Glinsky, T. M. O’Neill: Phys. Fluids B3 (1991) 1279.

[149] H. Friedrich, D. Wintgen: Phys. Repts. 183 (1989) 37.

[150] J. Main et al.: Phys. Rev. Lett. 57 (1986) 2789.

[151] G. Breit, E. Teller: Ap. J. 91 (1940) 215.

[152] [133], p. 197.

[153] [132], p. 285.

[154] M. Goeppert-Mayer: Ann. Phys. (Leipzig) 9 (1931) 273.

[155] D. Kleppner, in The Hydrogen Atom, G. F. Bassani, M. Inguscio, T. W. Hänsch, eds., Springer Verlag, Berlin, 1989, p. 103.

[156] A. Wolf: "Laser-stimulated radiative recombination", NATO Advanced Workshop on Recombination of Atomic Ions, Newcastle, Northern Ireland, 1991, HD-PY 92/05;

A. Wolf, In: Antihydrogen, Proc. Antihydrogen Workshop, Munich, 1992 (J. Eades, ed.); Hyp. Int. 76 (1993) 189.

[157] U. Schramm, J. Berger, M. Grieser, D. Habs, E. Jaeschke, G. Kilgus, D. Schwalm, A. Wolf, R. Neumann, R. Schuch, Phys. Rev. Lett. 67 (1991) 22.

[158] F. B. Yousif, P. Van der Donk, Z. Kucherovsky, J. Reis, E. Brannen, J. B. A. Mitchell, T. J. Morgan, Phys. Rev. Lett. 67 (1991) 26.

[159] R. Neumann, Hyp. Int. 44 (1988) 305.

[160] H. Poth et al., Hyp. Int. 44 (1988) 259;

H. Poth: Phys. Scripta 38 (1988) 806.

[161] J. W. Humbertson, M. Charlton, F. M. Jacobsen, B. I. Deutch, J. Phys. B: At. Mol. Phys. 20 (1987) L25.

[162] B. I. Deutch, L. H. Andersen, P. Hvelplund, F. M. Jacobsen, H. Knudsen, M. H. Holzscheiter, M. Charlton, G. Laricchia, Hyp. Int. 44 (1988) 271.

[163] B. I. Deutch, M. Charlton, M. H. Holzscheiter, P. Hvelplund, L. V. Jørgensen, H. Knudsen, G. Laricchia, J. P. Merrison, M. R. Poulsen, In: Antihydrogen, Proc. Antihydrogen Workshop, Munich, 1992 (J. Eades, ed.); Hyp. Int. 76 (1993) 153.

[164] M. Charlton, Phys. Lett. A143 (1990) 143. 
[165] G. Gabrielse, S. L. Rolston, L. Haarsma, W. Kells, Hyp. Int. 44 (1988) 287.

[166] W. Quint, R. Kaiser, D. Hall and G. Gabrielse, In: Antihydrogen, Proc. Antihydrogen Workshop, Munich, 1992 (J. Eades, ed.); Hyp. Int. 76 (1993) 181.

[167] C. H. Tseng, G. Gabrielse, In: Antihydrogen, Proc. Antihydrogen Workshop, Munich, 1992 (J. Eades, ed.); Hyp. Int. 76 (1993) 381.

[168] Ch. T. Munger, S. J. Brodsky, I. Schmidt, In: Antihydrogen, Proc. Antihydrogen Workshop, Munich, 1992 (J. Eades, ed.); Hyp. Int. 76 (1993) 175.

[169] M. Iwasaki, S. N. Nakamura, K. Shigaki, Y. Shimizu, H. Tamura, T. Ishikawa, R. S. Hayano, E. Takada, E. Widmann, H. Outa, M. Aoki, P. Kitching, T. Yamazaki, Phys. Rev. Lett. 67 (1991) 1246.

[170] T. Yamazaki, E. Widmann, R. S. Hayano, M. Iwasaki, S. N. Nakamura, K. Shigaki, F. J. Hartmann, H. Daniel, T. von Egidy, P. Hofmann, Y.-S. Kim, J. Eades, Nature 361 (1993) 238.

[171] T. Yamazaki: Z. Phys. A 341 (1992) 233.

[172] Y. Ito, E. Widmann and T. Yamazaki: In: Antihydrogen, Proc. Antihydrogen Workshop, Munich, 1992 (J. Eades, ed.); Hyp. Int. 76 (1993) 163.

[173] G. Torelli, N. Beverini, In Nuclear and Particle Physics at Intermediate Energies (T. Bressani and G. Pauli, eds.), Proc. Conf., Trieste, Italy, 1985, Vol. 3, p. 111.

[174] G.-Z. Li, R. Poggiani, G. Testera, G. Torelli, G. Werth, In: Antihydrogen, Proc. Antihydrogen Workshop, Munich, 1992 (J. Eades, ed.); Hyp. Int. 76 (1993) 343.

[175] R. Poggiani: In: Antihydrogen, Proc. Antihydrogen Workshop, Munich, 1992 (J. Eades, ed.); Hyp. Int. 76 (1993) 371.

[176] V. V. Parkhomchuk, Hyp. Int. 44 (1988) 315.

[177] J. T. M. Walraven, In: Antihydrogen, Proc. Antihydrogen Workshop, Munich, 1992 (J. Eades, ed.); Hyp. Int. 76 (1993) 205.

[178] G. Gabrielse, Hyp. Int. 44 (1988) 349.

[179] W. Ertmer, H. Wallis, Hyp. Int. 44 (1988) 319;

P. D. Lett, P. L. Gould, W. D. Phillips, Hyp. Int. 44 (1988) 335.

[180] J. M. Doyle, J. C. Sandberg, I. A. Yu, C. L. Cesar, D. Kleppner, T. J. Greytak, Phys. Rev. Lett. 67 (1991) 603.

[181] W. D. Phillips, S. L. Rolston, P. D. Lett, T. McIlrath, N. Vansteenkiste, C. I. Westbrook, In: Antihydrogen, Proc. Antihydrogen Workshop, Munich, 1992 (J. Eades, ed.); Hyp. Int. 76 (1993) 265.

[182] T. W. Hijmans, O. J. Luiten, I. D. Setija, J. T. M Walraven, J. Opt. Soc. Am. B6 (1989) 2235.

[183] I. D. Setija, H. G. C. Werij, O. J. Luiten, M. W. Reynolds, T. W. Hijmans, J. T. M. Walraven, Phys. Rev. Lett. 70 (1993) 2257.

[184] M. Weitz, F. Schmidt-Kaler, T. W. Hänsch, Phys. Rev. Lett. 68 (1992) 1120.

[185] P. Zhao et al.: Phys.Rev. A 39 (1989) 2888.

[186] D. H. McIntyre, W. M. Fairbanks, Jr., S. A. Lee, T. W. Hänsch, E. Riis, Phys. Rev. A 41 (1990) 4632.

[187] R. G. Hulet, D. Kleppner, Phys. Rev. Lett. 51 (1983) 1430.

[188] J. Hare, M. Gross, and P. Goy, Phys. Rev. Lett. 61 (1988) 1939. 